\title{
Exosomes from Human Umbilical Cord \\ Mesenchymal Stem Cells Facilitate Diabetic Wound Healing through miR-221-3p-mediated Enhancement of Angiogenesis
}

\section{Qian Wei}

Medical School of Chinese PLA: Chinese PLA General Hospital

Yaxi Wang

Medical School of Chinese PLA: Chinese PLA General Hospital

Kui Ma

Fourth Medical Center of PLA General Hospital

Xiaowei Bian

Fourth Medical Center of PLA General Hospital

Qiankun Li

Fourth Medical Center of PLA General Hospital

Bingmin Li

Fourth Medical Center of PLA General Hospital

Wenzhi Hu

Fourth Medical Center of PLA General Hospital

Haihong Li

Hubei University of Medicine

Xiaobing Fu

Fourth Medical Center of PLA General Hospital

Cuiping Zhang ( $\nabla$ zcp666666@sohu.com )

Forth Medical Center of General Hospital of PLA https://orcid.org/0000-0003-0320-6226

\section{Research}

Keywords: Diabetic wounds, Mesenchymal stem cells, Exosomes, MiR-221-3p/PTEN/AKT/HIF-1a/VEGF pathway, Angiogenesis

Posted Date: February 3rd, 2021

DOl: https://doi.org/10.21203/rs.3.rs-158498/v1 
License: (c) (i) This work is licensed under a Creative Commons Attribution 4.0 International License. Read Full License 


\section{Abstract}

Background: Endothelial dysfunction caused by persistent hyperglycemia in diabetes is responsible for impaired angiogenesis in diabetic wounds. Exosomes are considered potential therapeutic tools to promote diabetic wound healing. The aim of this study was to investigate the effects of exosomes secreted by human umbilical cord mesenchymal stem cells (hucMSC-Exos) on angiogenesis under high glucose $(\mathrm{HG})$ conditions in vivo and in vitro and to explore the underlying mechanisms.

Methods: HucMSC-Exos were used to treat diabetic wounds and human umbilical vascular endothelial cells (HUVECs) exposed to HG. Wound healing and angiogenesis were assessed in vivo. The biological characteristics of HUVECs were examined in vitro. Expression of pro-angiogenesis genes in HUVECs was also examined by western blotting. The miRNAs contained within hucMSC-Exos were identified using miRNA microarrays and qRT-PCR. The roles of selected miRNAs in angiogenesis were assessed using specific agomirs and inhibitors.

Results: In vivo, local application of hucMSC-Exos enhanced wound healing and angiogenesis. In vitro, hucMSC-Exos reduced senescence of HG-treated HUVECs and promoted proliferation, migration, and tube formation by inhibiting phosphatase and tensin homolog (PTEN) expression and activating the AKT/HIF1a/VEGF pathways. MiR-221-3p was enriched in hucMSC-Exos. In vitro, MiR-221-3p downregulated PTEN and activated the AKT/HIF-1a/VEGF pathway to promote proliferation, migration, and tube formation in HG-treated HUVECs. In vivo, miR-221-3p agomirs mimicked the effects of hucMSC-Exos on wound healing and angiogenesis, whereas miR-221-3p inhibitors reversed their effects.

Conclusions: Our findings suggest that hucMSC-Exos have regenerative and protective effects on HGinduced senescence in endothelial cells via transfer of miR-221-3p, thereby accelerating diabetic wound healing. Thus, hucMSC-Exos may be promising therapeutic candidates for improving diabetic wound angiogenesis.

\section{Introduction}

Diabetes is a multifaceted metabolic disorder. Nearly $20 \%$ of diabetics worldwide develop prolonged wounds, which may lead to the formation of skin ulceration [1]. Leg and foot ulcers are the most common types of diabetic wounds. Diabetic foot ulceration results in amputation in $15-27 \%$ of patients if not properly diagnosed and treated [2]. Despite improvements in wound repair, cure of chronic diabetic wounds remains a distant goal because of impaired healing. Angiogenesis plays a critical role in the wound healing process, facilitating the transport of nutrients and oxygen to lesion sites and enhancing fibroblasts multiplication, collagen synthesis and re-epithelization [3, 4]. Hypo-vascularization is associated with delayed or dysfunctional healing of diabetic wounds [5, 6]. Furthermore, hyperglycemia, a typical characteristic of diabetes, is commonly associated with refractory wounds and endothelial malfunction, which, in turn, lead to vascular complications such as diabetic foot ulceration [7]. 
Accordingly, novel remedies to promote angiogenesis would be highly valuable for managing diabetic wounds.

Mesenchymal stem cells (MSCs) have been shown to promote angiogenesis in ischemic conditions such as chronic wounds [8-10], myocardial infarction [11, 12], and cerebral ischemia [13, 14]. For example, human umbilical cord MSCs (hucMSCs) relieved hind limb ischemia by promoting angiogenesis in mice [15]. Recently, promotion of angiogenesis by MSCs has increasingly been attributed to exocrine factors $[16,17]$. Exosomes carry molecules including proteins, mRNAs, and miRNAs, and have been demonstrated to act as paracrine factors to regulate the interactions between MSCs and target cells. Compared with their parental MSCs, exosomes have several advantages including high stability, increased therapeutic efficiency and safety, absence of immune reactions, fewer ethical issues, and decreased potential for embolism formation and carcinogenicity [18-21]. Exosomes derived from many sources of MSCs have been reported to promote angiogenesis and represent promising treatment options for diabetic wounds. For instance, exosomes derived from bone marrow MSCs facilitated diabetic wound repair by increasing angiogenesis via the AKT/eNOS pathway [22]. Exosomes derived from hucMSCs (hucMSC-Exos) and lipopolysaccharide-preconditioned hucMSC-Exos regulated macrophage plasticity to resolve chronic inflammation during diabetic wound healing [23]. However, whether hucMSC-Exos can restore the proangiogenic ability of endothelial cells injured under high glucose (HG) conditions remains to be confirmed.

Exosomes contain multiple types of information-containing molecules including miRNAs, non-coding small RNAs that can bind to the 3'- untranslated region of mRNAs to inhibit target gene expression. Exosomal miRNAs have important regulatory functions and may participate in wound healing and angiogenesis. For example, exosomes derived from human adipose-derived MSCs transferred miRNA125 a to endothelial cells and promoted angiogenesis in vivo and in vitro by repressing Delta-like 4 expression [24]. Circulating exosomes containing miR-20b-5p isolated from diabetic patients slowed wound healing and angiogenesis by targeting Wnt9b/ $\beta$-catenin signaling [25]. Recently, hucMSC-Exos combined with pluronic F127 hydrogel were reported to have beneficial effects for diabetic wound treatment [26], but the mechanisms through which hucMSC-Exos exert their functions remain unclear.

In this study, we first assessed the effects of hucMSC-Exos on diabetic wound healing and angiogenesis. We used hucMSC-Exos to treat human umbilical vascular endothelial cells (HUVECs) exposed to HG. Subsequently, the proliferation, migration, tube formation, and senescence of HUVECs were examined. Expression of angiogenesis-related proteins including phosphatase and tensin homolog (PTEN), p-AKT, hypoxia inducible factor (HIF)-1a, and vascular endothelial growth factor (VEGF) was assessed. We also identified candidate miRNAs in hucMSC-Exos and showed that these could improve endothelial cell function and target the PTEN/AKT/HIF-1a/VEGF pathways.

\section{Methods}

\section{Culture and identification of hucMSCs}


The hucMSCs (P1) were acquired from the Chinese Academy of Sciences' cell bank and cultured in Dulbecco's modified Eagle's medium/F12 (DMEM/F12, Gibco, USA), containing 10\% Fetal Bovine Serum (Gibco, USA) and 1\% penicillin and streptomycin (Gibco, USA). The flow cytometer was employed to determine cell surface markers of hucMSCs. The hucMSCs were washed and co-incubated with fluorescence-conjugated antibodies (Abcam, USA) (CD73-PE, CD90-FITC, CD105-FITC, CD45-FITC, CD19FITC, HLA-DR-FITC and CD34FITC) at room temperature for three quarters of an hour. To identify the multi-differentiation potential, hucMSCs were incubated with chondrogenic, adipogenic and osteogenic differentiation medium (Cyagen, China), respectively. Next, these induced cells were stained severally with Alcian blue, Oil Red $\mathrm{O}$, and Alizarin Red S to evaluate chondrogenic, adipogenic, and osteogenic differentiation. Then these cell images were filmed with an Olympus IX71 light microscope (Olympus, Japan).

\section{Isolation and characteristics of hucMSC-Exos}

After 2-3 passages, the culture medium of hucMSCs was changed into DMEM/F12 containing 10\% exosome-free FBS (SBI, USA). The cultured supernatant of hucMSCs was collected every $72 \mathrm{~h}$ starting from passage 3 until passage 7. Exosomes were harvested from supernatant by means of a series of ultra-high-speed centrifugation procedures according to the protocol illustrated by Jia et al [27]. The characteristics of hucMSC-Exos were conducted by nanoparticle tracking analyzer (NTA) (Particle Metrix $\mathrm{GmbH}$, Germany) and transmission electron microscope (TEM) (Hitachi, Japan). Expressions of the hucMSC-Exos markers containing CD81, CD63, CD9, the tumor susceptibility gene101 (TSG101), were examined by Western blot.

\section{In vivo administration of hucMSC-Exos}

All animal procedures involved were approved by the Animal Research Committee of PLA General Hospital in Beijing, China and were conducted in accordance with the principles and procedures of the National Institutes of Health $(\mathrm{NIH})$ Guide for the Care and Use of Laboratory Animals. Remarkable efforts were made to minimize the number of animals and their sufferings. Thirty male diabetic mice of 8 weeks (BKS-Dock Lepr ${ }^{\mathrm{em} 2 \mathrm{Cd} 479}, \mathrm{db} / \mathrm{db}$ ), weighing about $35 \mathrm{~g}$, were anesthetized with $4 \%$ chloral hydrate. Fullthickness skin wounds of $18 \mathrm{~mm}$ in diameter were created on the backs of diabetic mice. Afterward, all mice were randomly divided into exosome groups $(7,14$, and 21 days) and control groups $(7,14$, and 21 days). There were five mice for each time point. HucMSC-Exos $(100 \mathrm{~mL}, 200 \mathrm{ug} / \mathrm{mL})$ and PBS $(100 \mathrm{~mL})$ were injected around the wounds at 4 sites ( $25 \mu \mathrm{L}$ per site) with a micro syringe (Hamilton) at every other day.

\section{Wound closure evaluation and neovascularization observation}


Wounds from each group were imaged at $0,7,14$ and 21 days post-wounding. All wounds were calculated with a caliper ruler and areas of them were evaluated in Image -Pro Plus software (Media Cybernetics, USA). The wound closing rate was calculated as the following formula: $\left(A_{0}-A_{t}\right) / A_{0} \times 100 \%$. $A_{0}$ represents the primary wound area and $A_{t}$ represents the wound area at 7,14 and 21 days. To detect the generation of new blood vessels, the skin underside at day 21 was observed and captured using a digital camera and a microscope at $2.5 \times$ magnification. Percentage of vessel area was measured as previously described [22].

\section{Histological analysis and immunohistochemistry}

After the mice were put to euthanasia by being excessively injected with pentobarbital, wound sites were obtained and fixed postoperatively. The obtained tissues were dehydrated step and step and embedded in paraffin. Subsequently, these embedded tissues were cut into $5 \mu \mathrm{m}$ thick sections and stained with hematoxylin and eosin (H\&E). Measurement of scar width and neo-epithelium percentage was performed as previously described [28]. Masson's trichrome was stained to assess the extent of collagen maturity. Collagen volume fraction were quantified according to literature previously described [29].

Immunohistochemistry staining for CD31 (Abcam, the USA, ab28364, 1:100) was conducted to examine the degree of newly-formed capillaries in wound sites. Briefly, the paraffin sections were incubated with the primary CD31 antibody overnight at $4{ }^{\circ} \mathrm{C}$ and then with the secondary HRP-conjugated antibody (1:200, Abcam) for $1 \mathrm{~h}$ at room temperature. Finally, the sections were colored by a DAB kit (ZSGB-BIO, China). Images were photographed under a microscope and measured as literature previously described [30].

\section{Blood perfusion evaluation}

On day 21 postoperation, we applied laser speckle contrast imaging (LSCl) to investigate the wound perfusion. A PeriCam PSI-ZR (PERIMED Ltd, Sweden) was employed to record images of each wound. A unit of perfusion was detected based on a near-infrared laser $(785 \mathrm{~nm})$ for perfusion measurements. Images were captured using the same scan site dimensions at a fixed distance from the wound surface. Applying PIMSoft (Moor Instruments Ltd, UK), flux photographs of each wound were estimated to evaluate the mean perfusion units (MPUs) ratio calculated by comparing the MPUs at the wound site (ROI-1) with that of the area besides the wound (ROI-2).

\section{Cell culture and hucMSC-Exo uptake}

HUVECs were obtained from the Chinese Academy of Sciences (Shanghai, China). These cells were incubated in Dulbecco's modified Eagle's medium (DMEM, Gibco, USA) consisting of $10 \%$ fetal bovine serum (Gibco, USA) and 1\% penicillinstreptomycin. The normal glucose (NG) concentration is $5.5 \mathrm{mM}$. To 
examine hucMSC-Exo uptake by HUVECs, exosomes were labeled with PKH67 (Sigma-Aldrich, Germany), a green fluorescent dye, in the light of the manufacturer's protocol. Then, HUVECs were co-cultured with PKH67-labeled exosomes for $4.5 \mathrm{~h}$ and next fixed in $4 \%$ paraformaldehyde for $15 \mathrm{~min}$. After these cells were washed by PBS, HUVECs were incubated with CD31 antibody $(1: 100)$ overnight at $4{ }^{\circ} \mathrm{C}$. Next Cy3conjugated secondary antibody (Abcam, 1:500) and DPAI (Sigma-Aldrich, $2 \mathrm{mg} / \mathrm{mL}$ ) were applied for the incubation for visualization. At last, HUVECs were observed via a confocal microscope (Leica, Germany).

\section{Proliferation and cell cycle assay}

For proliferation assay, HUVECs $\left(8 \times 10^{3}\right.$ cells per well) were seeded into 96 -well plates. The cells in control group were cultured in NG concentration and those in HG group were incubated with glucose concentration of $35 \mathrm{mM}$. Different concentrations of exosomes $(50,100,150,200 \mu \mathrm{g} / \mathrm{mL})$ were added to the culture medium of HG-induced HUVECs to select the optimal concentration. On day 1,3,5 and 7, cell counting kit-8 (CCK-8, $10 \mu \mathrm{L}$ per well, Dojindo Molecular Technologies, Japan) was added to the medium ( $100 \mu \mathrm{L}$ per well). The absorbance of per well was examined at $450 \mathrm{~nm}$ by an enzyme immunoassay analyzer (Bio-Rad 680, Hercules, USA) after incubation for $3 \mathrm{~h}$ at $37^{\circ} \mathrm{C}$. According to the results of CCK-8 assay, the optimal concentration of exosomes, which had the most obvious effect on cell viability, was chosen to applied in the following experiments including cell cycle, cell migration, tube formation, and cellular senescence assays. Additionally, the expression of PTEN, p-AKT, AKT, HIF-1a, and VEGF was evaluated by Western blot.

For cell cycle assay, HUVECs $\left(8 \times 10^{5}\right.$ cells per well) were seeded in six-well plates and cultured in NG, HG and $\mathrm{HG}+$ Exos. After being cultured for 3 days, the cells were harvested from six-well plates and fixed with $70 \%$ cold ethanol overnight at $4{ }^{\circ} \mathrm{C}$. On the second day, single cell suspensions were put into digestion with $100 \mu \mathrm{l} /$ well DNase-free RNase in incubator at $37^{\circ} \mathrm{C}$, and subsequently $400 \mu \mathrm{l}$ of propidium iodide (PI) solution was added for DNA staining for $1 \mathrm{~h}$ at $4{ }^{\circ} \mathrm{C}$. PI fluorescence as well as forward light scattering was examined with a flow cytometer (BD FACS Calibur ${ }^{\mathrm{TM}}$, Becton-Dickinson, USA). The percent of cells in different phases was calculated.

\section{Cell migration assay}

The migration property of HUVECs was evaluated by means of scratch and transwell assay. For scratch assay, HUVECs were cultured in six-well plates. When $90 \%$ confluence was reached, cells were scratched with a pipette tip. At planned time point, cells were photographed using a microscope. Distances between two borders of the scratch were calculated by ImageJ software. The migration rate (\%) was calculated as: $\left(M_{0}-M_{t}\right) / M_{0} \times 100 \%$, in which $M_{0}$ stood for the initial scratch distances, while Mt represented the resting scratch distances at the surveyed time point. For transwell assay, approximate $0.5 \times 10^{4} \mathrm{HUVECs}$ were suspended in serum-free medium and seeded into the upper chamber of 24-well plates (Corning) with $8.0 \mu \mathrm{m}$ polycarbonate membrane. Then the complete medium (containing serum) supplemented 
with $\mathrm{HG}$ or Exos was added into the lower chamber. After $24 \mathrm{~h}$, we swabbed the cells on the upper surface of the filter membranes and the migrated cells on the lower surface were stained with crystal violet (Solarbio, $0.1 \%, w / v)$ for $7 \mathrm{~min}$. The stained cells were observed and calculated under an optical microscope.

\section{Tube formation assay}

$50 \mu \mathrm{l}$ cold Matrigel was added into each well of a pre-cooling 96 -well plate and next incubated at $37^{\circ} \mathrm{C}$ for 30 min. HUVECs $\left(2 \times 10^{4}\right.$ cells per well, five replicates per group) were put into the Matrigel-coated plates and treated with different mediums (NG, HG and HG + Exos). After incubation for $6 \mathrm{~h}$ at $37{ }^{\circ} \mathrm{C}$, tube formation was examined under a microscope. The parameters (total branching points and total tube length) demonstrating the tube formation capability were measured with Image-Pro Plus 6.0 software.

\section{Cellular senescence assay}

Reactive oxygen species (ROS) measurement, SA- $\beta$-gal staining and the detection of age-related genes including p21, p16 and p53 were performed to evaluate cellular senescence. After cultured under design conditions (NG, HG and HG + Exos), HUVECs were incubated with $10 \mu \mathrm{M}$ 2',7'-dichlorodihydrofluorescein diacetate (DCFDA, Sigma-Aldrich, Germany). The accumulation of ROS in cells was quantified using flow cytometer (BD FACS Calibur ${ }^{\text {TM }}$, Becton-Dickinson, USA). SA- $\beta$-gal staining was conducted with a SA- $\beta$-gal staining kit in accordance with the manufacturer's protocols. Briefly, HUVECs were washed three times with PBS and then fixed with $4 \%$ paraformaldehyde for $30 \mathrm{~min}$. After incubated with staining solution overnight under $37^{\circ} \mathrm{C} \mathrm{CO}_{2}$-free condition, the cells were observed with a microscope. The ratio of SA- $\beta$-gal positive cells was calculated by counting blue cells versus total cells. The expression of age-related genes was performed by Western blot.

\section{PTEN siRNA interference}

To assess whether the knocking down of PTEN expression can achieve the similar effects of hucMSCExos on HG-induced HUVECs, PTEN siRNAs were obtained from RiboBio (Guangzhou, China) and used to knock down the expression of PTEN in HUVECs. Briefly, HG-induced HUVECs were transfected with siRNAs or the universal negative control siRNA (Con siRNA) using Lipofectamine 2000 according to the manufacturer's instructions. After $24 \mathrm{~h}$, the expression of PTEN was detected by qRT-PCR to evaluate the inhibitory efficiency of siRNAs. In addition, the proliferation of HUVECs was evaluated by CCK-8 at day 1 , 3,5 and 7 . Then we performed the downstream experiments including scratch, transwell, and tube formation assays (the methods are the same as above).

\section{Detection of miRNAs in hucMSC-Exos}


MiRNA microarray and qRT-PCR were perform to find the candidate exosomal miRNAs which play an important role in hucMSC-Exos-induced effects. MiRNAs in exosomes were isolated via the use of the SeraMir Exosome RNA Purification Kit (System Biosciences, Mountain View, USA). MiRNA microarray was conducted using GeneChip miRNA 4.0 array which contains 30424 probe sets covering 2578 human miRNAs, 728 rat miRNAs, and 1908 mouse miRNAs. The miRNAs highly expressed in hucMSC-Exos were further confirmed by qRT-PCR. Bioinformatics analysis was conducted by programs including Targetscan (http://www.targetscan.org/), miRanda (http://www.microrna.org), and Pictar (http://pictar.mdcberlin.de/). According to the results of miRNA detection and bioinformatics analysis, miR-221-3p was found to be the most highly expressed miRNA and target PTEN. Next, the potential role of miR-221-3p in hucMSC-Exos was explored.

\section{MiRNA interference}

We obtained miR-221-3p inhibitors, negative control (NC) inhibitors, miR-221-3p agomirs, and negative control agomirs from GenePharma (Suzhou, China). To produce exosomes without miR-221-3p, miR-221$3 p$ inhibitors or $\mathrm{NC}$ inhibitors $(100 \mathrm{nmol} / \mathrm{L})$ were transfected into hucMSCs which have grown to $80 \%$ confluence with aid of Lipofectamine 2000 (Invitrogen, NY, USA). After $6 \mathrm{~h}$, the transfected cells were maintained in the medium containing Exos-free FBS for $48 \mathrm{~h}$. Exosomes were isolated from the conditioned medium of the cells transfected with miR-221-3p inhibitor or NC inhibitor. These exosomes were named as Exos-inhibitor miR-221-3p (exosomes without miR-221-3p) and Exos-inhibitor ${ }^{N C}$, respectively. Next, HG-treated HUVECs were incubated with Exos-inhibitor miR-221-3p or Exos-inhibitor ${ }^{N C}$, or transfected with miR-221-3p agomirs or NC agomirs. The expressions of miR-221-3p and PTEN in HUVECs from different groups were detected by qRT-PCR. We also observed the expressions of PTEN, pAKT, AKT, HIF-1 a, and VEGF by Western blot. The proliferation, migration and tube formation abilities were investigated by the methods described above. Additionally, animal experiment with mice was also performed to evaluate the potential role of miR-221-3p in hucMSC-Exos. The experiment designs the groups as in the cellular experiments. Wound closure, blood perfusion and angiogenesis were examined by the methods in above animal experiment.

\section{Western blot}

The total protein was harvested using RIPA buffer with a protease phosphatase inhibitor mixture (Solarbio, China). 10\% SDS-PAGE was employed to separate proteins. The PVDF membrane (Millipore) was utilized to blot the proteins. Skimmed milk was applied to block the membranes for $1 \mathrm{~h}$. Next, the PVDF membranes were incubated with primary antibodies including anti-CD81, anti-CD63, anti-TSG101, anti-CD9, anti-p21, anti-p53, anti-p16, anti-AKT, anti-phosphorylate AKT (anti-p-AKT), anti-PTEN, antiHIF1a, and anti-VEGF (Abcam, USA) at $4{ }^{\circ} \mathrm{C}$ overnight followed by being treated with horseradish peroxidase-conjugated (HRP)-linked secondary antibodies (ZSGB-BIO, China) for $1 \mathrm{~h}$ at room temperature on the next day. The immunoreactive bands were visualized via an ECL kit (Solarbio, China) and imaged 
by UVITEC Alliance MINI HD9 system (UVITEC, Britain). The protein expression level was quantified by ImageJ.

\section{qRT-PCR analysis}

Total RNA was extracted from HUVECs with Trizol Reagent (Invitrogen, USA) and cDNA was synthetized out of $1 \mu \mathrm{g}$ of total RNA by employing the Revert Aid first-strand cDNA synthesis kit (Fermentas, Life Sciences, Canada). Next, qRT-PCR was conducted with an ABI PRISM®7900HT System with SYBR Premix ExTaqTM II (Takara Biotechnology, Japan). GAPDH was applied to normalize the results. Primer sequences used for qRT-PCR were seen in Table S1. For detection of miRNAs, exosomal miRNAs were isolated via the use of the SeraMir Exosome RNA Purification Kit (System Biosciences, Mountain View, USA), and cDNA for miRNAs was integrated with TaqMan microRNA assay kit (Applied Biosystems, Foster City, USA) according to the manufacturer's protocol. The qRT-PCR reaction was conducted using FastStart Universal SYBR Green Master Mix (Roche, Indianapolis, USA) with the miRNA-specific forward primer (Yanzai Biotech, Shanghai, China; Table S1) and the universal reverse primer produced by the TaqMan miRNA assay kit. U6 small nuclear RNA was for normalization.

\section{Statistical analysis}

All data were reported as mean \pm standard deviation (SD) and evaluated with analysis of variance (ANOVA) and Student $t$ test where appropriate. All experiments were carried out at least three times. Differences were considered to be statistically significant when $p<0.05$.

\section{Results}

\section{HucMSC-Exos enhance cutaneous wound healing in diabetic mice}

HucMSCs were cultured and identified based on their morphology, phenotype, and function (Figure S1). Exosomes were isolated from hucMSCs and characterized by NTA, TEM and western blotting. NTA showed that the sizes of hucMSC-Exos ranged from $60 \mathrm{~nm}$ to $180 \mathrm{~nm}$ (mean diameter $100 \mathrm{~nm}$ ), whereas the concentration of exosomes was $8.77 \times 10^{9}$ particles $/ \mathrm{mL}$ (Fig. 1A). TEM revealed that hucMSC-Exos had a "saucer-like" ultrastructure (Fig. 1B). Representative exosomal markers including TSG101, CD63, CD9 and CD81 were expressed by the hucMSC-Exos (Fig. 1C). These results, which were consistent with those of previous reports [31-33], demonstrated that the isolated nanoparticles were indeed exosomes.

To evaluate the effects of hucMSC-Exos on diabetic wound healing, we created full-thickness cutaneous wounds on the backs of diabetic mice and injected hucMSC-Exos or phosphate-buffered saline (PBS) around the wounds. Digital photographs of the wound sites taken at day 0, 7, 14, and 21 showed the progress of wound closure in different groups of mice. Gross observations showed that the hucMSC-Exo- 
treated mice had reduced wound areas at days 7, 14, and 21 compared with PBS-treated control mice (Fig. 1D). The wounds of hucMSC-Exo-treated mice had mostly recovered at day 21, whereas scar areas remained discernable in the control mice. The dimensions of the wound areas were measured $0,7,14$, and 21 days after wounding. As shown in Fig. 1E, the extent of wound healing in hucMSC-Exo-treated mice was significantly greater after 7,14 , and 21 days than that in control mice $(p<0.001)$. At 21 days after wounding, wounds in hucMSC-Exo-treated mice were completely healed, whereas those of control mice still had approximately $40 \%$ of their area remaining to be closed. Rapid reepithelialization is one of the key steps in wound healing. Consistent with the wound area observations, hematoxylin and eosin staining showed that hucMSC-Exo-treated wounds had longer neo-epidermis and dermis lengths than those of PBS-treated wounds at day 7,14 and 21 post-wounding (Fig. 1F). Furthermore, quantitation of re-epithelialization rates and scar widths showed that hucMSC-Exo treatment facilitated epidermal regeneration and diminished scar formation at wound sites (Fig. S2). Masson staining also revealed a greater number of wavy fibers in exosome-treated wounds compared with control wounds (Fig. 1G). At the end 21 days, the collagen volume fractions of exosome-treated and control wounds were $70.13 \pm 5.76$ $\%$ vs. $43.20 \pm 7.70 \%(p<0.001)$. These data suggested that hucMSC-Exo treatment can promote wound healing in diabetic mice.

\section{HucMSC-Exos promote angiogenesis in the wound sites of diabetic mice}

We next assessed whether treatment with hucMSC-Exos could promote angiogenesis at wound sites, thereby enhancing diabetic wound healing. As shown in Fig. 2A, larger amounts of newly formed blood vessels were observed in hucMSC-Exo-treated wounds at day 21 post-wounding compared with PBStreated wounds. The percentage vessel area in hucMSC-Exo-treated wounds was significantly higher than that in PBS-treated wounds ( $41.01 \pm 2.43 \%$ vs. $20.01 \pm 7.96 \%$; $<0.001)$ (Fig. 2B). The blood flow at wound sites in different groups was evaluated by small animal doppler. Flux images of hucMSC-Exotreated wounds showed larger red areas than control wounds (Fig. $2 \mathrm{C} \mathrm{left),} \mathrm{reflecting} \mathrm{better} \mathrm{blood}$ perfusion in hucMSC-Exo-treated wounds. The mean perfusion unit (MPU) ratio in hucMSC-Exo-treated mice was also higher than that in control mice ( $2.57 \pm 0.44$ vs. $1.64 \pm 0.24 ; p<0.01)$ (Fig. $2 \mathrm{C}$ right). CD31 is a marker for newly formed blood vessels. Immunohistochemistry for CD31 (Fig. 2D) revealed that newly developed blood vessels at wound sites were increased following treatment with hucMSC-Exos compared with controls from day $7(0.28 \pm 0.06$ vs. $0.14 \pm 0.01 ; p<0.05)$ to day $14(0.57 \pm 0.02$ vs. $0.19 \pm$ $0.01 ; p<0.001$ ) (Fig. 2E). Collectively, these findings suggested that hucMSC-Exos can promote angiogenesis at wound sites in diabetic mice.

\section{HucMSC-Exos improve the function of HG-treated HUVECs in vitro}


To understand the multiple effects of hucMSC-Exos on HUVECs, we first determined whether hucMSCExos could be internalized into endothelial cells. Previous studies illustrated that adhesion molecules on the surface of exosomes are associated with adherence to specific cells, but the cellular and molecular bases for specific targeting of recipient cells remain unclear. Therefore, we tested whether hucMSC-Exos could be taken up by HUVECs. HucMSC-Exos were labeled with PKH67 and then incubated with HUVECs. Next, recipient HUVECs were washed with PBS to remove unbound labeled exosomes. After fixation with paraformaldehyde, recipient cells were stained with CD31 (red) and 4',6-diamidino-2-phenylindole. As shown in Fig. 3A, all HUVECs were stained green after incubation with labeled hucMSC-Exos for $4.5 \mathrm{~h}$, demonstrating that PKH67-tagged hucMSC-Exos had been transferred to the perinuclear regions of HUVECS.

To simulate a HG microenvironment in vitro, HUVECs were treated with D-glucose at a final concentration of $35 \mathrm{mM}$ as previously described [34]. The results of a cell counting kit-8 (CCK-8) assay showed lower proliferation of HG-treated HUVECs compared with normal glucose (NG)-treated HUVECs (Fig. 3B). Different concentrations of exosomes were added to the culture medium of HG-induced HUVECs. Treatment with hucMSC-Exos significantly enhanced the viability of HG-induced HUVECs at days 3, 5 and 7 in a dose-dependent manner (Fig. S3). The $200 \mu \mathrm{g} / \mathrm{mL}$ concentration of hucMSC-Exos had the greatest impact on cell viability (Fig. 3B). This result was further confirmed using a cell cycle assay. The proportions of three cellular subpopulations $\left(G_{0} / G_{1}, S\right.$ and $\left.G_{2} / M\right)$ were estimated from DNA distribution as determined by flow cytometry. $\mathrm{HG}$ stimulation inhibited the $\mathrm{G}_{1}-\mathrm{S}$ transition, and treatment with hucMSC-Exos partially rescued HG-induced cell cycle arrest. In HG + hucMSC-Exo-treated HUVECs, more cells were in the $S$ and $G_{2} / M$ phases compared with HUVECs treated with $H G$ alone ( $33.30 \pm 2.98 \%$ vs. $16.55 \pm 1.89 \% ; p<0.0001$ ), suggesting that hucMSC-Exos promoted entry into the proliferative phase (Fig. 3C, D). Migration of HUVECs was evaluated using wound scratch and transwell assays. Scratch assays demonstrated that the migration rates of HG-induced HUVECs were decreased significantly compared with NG-induced HUVECs (migrating area of $24 \mathrm{~h}: 37.70 \pm 2.50 \%$ vs. $86.88 \pm 3.17 \%$; $<<0.01$ ). However, hucMSC-Exo stimulation improved the migration rates of HG-treated HUVECs (migrating area of 24h: $65.20 \pm 3.13 \%$ vs. $37.70 \pm 2.50 \%$; $p<0.01$ ) (Fig. 3E, F). Consistent with the results of the scratch assay, the number of migrated HG-treated HUVECs was less than the number of migrated NG-treated HUVECs (147.80 \pm 8.13 vs. $404.4 \pm 12.80 ; p<0.0001)$. Following treatment with hucMSC-Exos, the number of migrated cells was increased (353.40 \pm 8.71 vs. $147.80 \pm 8.13 ; p<0.0001)$ (Fig. 3G, H). The tube formation assay conducted on Matrigel is a model of angiogenesis in vitro. As shown in Fig. $3 \mathrm{l}$, the number of capillary-like structures was decreased in HG-treated HUVECs compared with NG-treated HUVECs $(23.60 \pm 4.08$ vs. $119.40 \pm 9.41 ; p<0.0001)$. The number of capillary-like structures formed by HG-treated HUVECs increased after incubation with hucMSC-Exos (58.20 \pm 3.19 vs. $23.60 \pm 4.08 ; p<$ 0.0001). Quantitative measurements showed that total branch points and total branching length were significantly increased after incubation of HG-treated HUVECs with hucMSC-Exos (Fig. 3J). These results indicated that hucMSC-Exos promoted proliferation, migration, and tube formation in HG-treated HUVECs. 
We also investigated whether hucMSC-Exos could mitigate endothelial cell senescence induced by HG. As potent oxidizing agents, reactive oxygen species (ROS) can directly cause cell senescence. Intracellular ROS levels in HUVECs are shown in Fig. S4. Higher amounts of ROS were detected in HG-treated HUVECs compared with NG-treated HUVECs. However, ROS levels were significantly decreased in hucMSC-Exotreated HG-induced HUVECs. To confirm the effects of hucMSC-Exos on cell senescence, we performed a senescence-associated $\beta$-galactosidase (SA- $\beta$-gal) assay on HG-treated HUVECs with or without exosome treatment. Consistent with analyses of ROS generation, expression of SA- $\beta$-gal in HG-treated HUVECs was reduced following exosome treatment (78.20 $\pm 4.41 \%$ vs. $20.60 \pm 2.21 \% ; p<0.0001)$ (Fig. S5). The expression levels of p21, p53 and p16 were examined by western blotting; these proteins are representative effectors and/or markers of cell senescence. As shown in Fig. S6, expression levels of p21, p53 and p16 were upregulated in HG-treated HUVECs but down-regulated following incubation of these cells with hucMSC-Exos. Thus, hucMSC-Exos block the p21, p53 and p16 signaling pathways. Collectively, these results showed that hucMSC-Exos could antagonize senescence in HG-induced HUVECS.

\section{HucMSC-Exos regulate PTEN/AKT/HIF-1a/VEGF signaling pathway}

Next, we explored the underlying mechanisms through which hucMSC-Exos inhibited senescence of HGtreated HUVECs. Previous studies showed that the PTEN/AKT/HIF-1a/VEGF pathways played important roles in processes related to angiogenesis, including proliferation, migration and tube formation of endothelial cells [35]. Thus, we assessed the expression of PTEN, p-AKT, AKT, HIF-1a, and VEGF in HUVECs using western blotting (Fig. 4A). PTEN is an upstream regulator of the AKT/ HIF-1a/VEGF pathways, restricting cell growth and motility signaling [36] by inhibiting phosphorylation of AKT. Western blotting showed that HG significantly increased the expressions of PTEN in HUVECs $(p<0.0001)$, whereas hucMSC-Exo treatment reversed this effect $(p<0.0001)$ (Fig. 4B). As expected, the ratio of $p$-AKT

to AKT was decreased by HG treatment compared with NG treatment $(p<0.0001)$ but increased following hucMSC-Exo treatment of HG-induced HVECs $(p<0.001)$ (Fig. 4C). It was reported that phosphorylation of AKT can increase the expression of HIF-1 $a$ and that VEGF is the most important proangiogenic target of HIF-1 a [37]. Consistent with these findings, we observed increased expression of HIF-1a and VEGF in HG-induced HUVECs after incubation with hucMSC-Exos ( $p<0.01$ ) (Fig. 4D, E). From the above results, we concluded that hucMSC-Exos can activate the PTEN/AKT/HIF-1a/VEGF signaling pathways, which may be responsible for the functional improvements of HG-treated HUVECs.

\section{PTEN knockdown generates hucMSC-Exos-like beneficial effects on HG-treated HUVECs}

We then asked whether silencing of PTEN expression could achieve similar effects to those of hucMSCExo treatment in HG-induced HUVECs. PTEN siRNAs (siPTEN) were used to silence the expression of 
PTEN. HG-induced HUVECs were divided into three groups: control, hucMSC-Exo-treated, and siPTENtreated. The inhibitory effect of the siRNAs was confirmed by qRT-PCR. As shown in Fig. 5A, PTEN expression was decreased by treatment with PTEN siRNAs to levels comparable to those in hucMSC-Exotreated HUVECs. CCK-8 assays showed that both hucMSC-Exos and PTEN siRNA significantly increased the proliferative capacity of HG-induced HUVECs (Fig. 5B). Additionally, the cellular migration area was significantly increased in both hucMSC-Exos-treated $(p<0.0001)$ and siPTEN-treated $(p<0.001)$ HUVECs compared with control HUVECs (Fig. 5C, D). This effect was further confirmed using the transwell assay. The numbers of migrated hucMSC-Exos-treated HUVECs $(p<0.0001)$ and siPTEN-treated HUVECs $(p<$ 0.001) were significantly enhanced compared with control HUVECs (Fig. 5E, F). Tube formation assays also demonstrated that PTEN siRNAs partially mimicked the effect of hucMSC-Exos on tube formation in HG-induced HUVECs (Fig. 5G, H). Taken together, these data demonstrated that the PTEN/AKT/HIF$1 a /$ VEGF signaling pathways played crucial roles in the improvement of endothelial cell function by hucMSC-Exos.

\section{hucMSC-Exo miR-221-3p downregulates PTEN and activates the AKT/HIF-1a/VEGF pathways in HG-treated HUVECs}

Exosomes contain a large number of information-containing molecules including miRNAs that can be transferred to target cells to modulate cellular function [38]. We hypothesized that miRNAs in hucMSCExos may be conveyed into HUVECs to regulate PTEN/AKT/HIF-1a/VEGF signaling and improve the function of HG-treated HUVECs. To explore the miRNAs within hucMSC-Exos that may be involved in angiogenesis, we examined exosomal miRNA expression using a miRNA microarray. The four most abundant miRNAs in hucMSC-Exos (miR-221-3p, miR-3960, miR-4497, and miR-6087-5p) accounted for $27.9 \%$ of total miRNA reads (Fig. 6A). We selected a group of miRNAs for further characterization (miR21-3p/5p, miR-221-3p/5p, miR-125a-5p/3p, miR-19a-5p/3p, miR-27a-3p/5p and miR-214-5p/3p); these miRNAs were reported to play roles in regulating endothelial cell function in vitro and/or angiogenesis in vivo. qRT-PCR confirmed the presence of the selected miRNAs in hucMSC-Exos. Consistent with the results of miRNA microarrays, miR-221-3p had the highest expression among the detected microRNAs (Fig. 6B). We investigated the potential targets of miR-221-3p by searching Targetscan, miRanda and Pictar. Among the candidate targets, the 3 '-untranslated region of human PTEN contained a putative region (nucleotides 200-206, NM_000314) matching the seed sequence of miR-221-3p that was conserved in rats and mice (Fig. 6C). Although studies of miR-221 in tumor development abound [39-43], studies of the role of this miRNA in wound healing are scarce [22,44]. Therefore, we focused on exosomal miR-221-3p for further experiments.

To verify the transfer of miR-221-3p into target cells, HG-treated HUVECs were co-cultured with PBS or hucMSC-Exos. As shown in Fig. 6D, miR-221-3p levels were significantly increased after cells were cocultured with hucMSC-Exos for $4.5 \mathrm{~h}$ compared with PBS-treated cells $(\mathrm{p}<0.01)$. After $24 \mathrm{~h}$, expression 
levels of PTEN were remarkably reduced in hucMSC-Exos-treated HUVECs $(p<0.001)$ (Fig. 6E). These results confirmed that miR-221-3p in hucMSC-Exos can be transferred into HG-treated HUVECs and that PTEN was targeted by miR-221-3p. Next, we explored whether miR-221-3p targeting of PTEN activated AKT/HIF-1a/VEGF signaling. First, we produced exosomes lacking miR-221-3p by transfecting hucMSCs with miR-221-3p inhibitors [45]. Exosomes isolated from hucMSCs transfected with miR-221-3p inhibitors were named Exos-inhibitor ${ }^{m i R-221-3 p}$ and those isolated from hucMSCs transfected with negative control (NC) inhibitors were named Exos-inhibitor ${ }^{\mathrm{NC}}$. Exos-inhibitor ${ }^{\mathrm{miR}-221-3 \mathrm{p}}$ or Exos-inhibitor ${ }^{\mathrm{NC}}$ were used to treat HG-induced HUVECs, and cells were transfected with miR-221-3p agomirs or NC agomirs induced with HG. qRT-PCR analysis showed that miR-221-3p expression was decreased in Exos-inhibitor ${ }^{\text {miR-221-3p_ }}$ treated HUVECs compared with Exos-inhibitor ${ }^{N C}$-treated HUVECs $(p<0.05)$, whereas a significant increase in miR-221-3p expression in miR-221-3p agomir-transfected cells, but not in NC agomirtransfected cells $(p<0.001$ ) (Fig. 6F), was found. As expected, hucMSC-Exos lacking miR-221-3p did not suppress PTEN mRNA expression. By contrast, miR-221-3p agomirs significantly suppressed PTEN mRNA levels $(p<0.0001)$, similar to the effects of hucMSC-Exos (Fig. 6G). Furthermore, protein expression of PTEN, p-AKT, AKT, HIF-1a, and VEGF was also assessed by western blotting and showed results consistent with mRNA levels (Fig. $6 \mathrm{H}, \mathrm{I}$ ). Thus, hucMSC-Exos treatment promoted the expression of $p$-AKT, HIF-1a and VEGF ( $<<0.0001)$, whereas their up-regulation was reduced in Exos-inhibitor ${ }^{\text {miR-221- }}$ $3 p$-treated HUVECs $(p<0.001)$. Exosome-like up-regulation could be induced by a miR-221-3p agomir. Collectively, these data demonstrated that miR-221-3p in hucMSC-Exos could activate AKT/HIF-1a/VEGF signaling by down-regulating PTEN expression.

\section{MiR-221-3p in hucMSC-Exos promotes proliferation, migration and tube formation of HG-treated HUVECs}

We also investigated the proliferation, migration, and tube formation of HG-treated HUVECs treated or transfected with NC agomirs, Exos-inhibitor ${ }^{N C}$, Exos-inhibitor ${ }^{\text {miR-221-3p }}$, and miR-221-3p agomirs. CCK-8 assays revealed that Exos-inhibitor ${ }^{N C}$ treatment and miR-221-3p agomir transfection significantly enhanced the proliferative capacity of HG-induced HUVECs (Fig. 7A). The miR-221-3p agomir partially mimicked the effects of Exos-inhibitor ${ }^{N C}$ on the proliferation of HG-induced HUVECs, indicating that other molecules within hucMSC-Exos may be involved in regulating the cellular function of HG-induced HUVECs (Fig. 7A). Next, the wound scratch and transwell assays were used to evaluate the properties of HG-induced HUVECs treated or transfected with exosomes or miRNA agomirs. The cellular migration area was significantly increased in Exos-inhibitor ${ }^{N C}$-treated HUVECs $(p<0.0001)$, whereas Exos-inhibitor ${ }^{m i R-}$ 221-3p treatment showed no such effect. MiR-221-3p agomirs partially mimicked the effect Exosinhibitor ${ }^{N C}$ in enhancing cellular migration $(p<0.0001)$ compared with $\mathrm{NC}$ agomirs (Fig. 7B, C). A transwell assay further confirmed these effects on cell migration. The numbers of migrated Exosinhibitor ${ }^{N C}$-treated HUVECs $(p<0.0001)$ and miR-221-3p agomir-transfected HUVECs $(p<0.0001)$ were

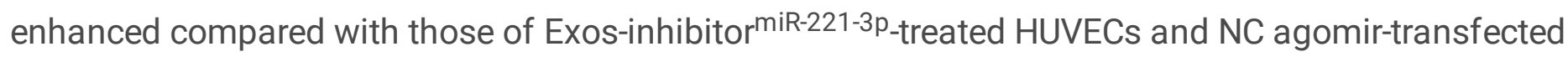


HUVECs (Fig. 7D, E). The total branching points and total tube length were calculated to evaluate the angiogenic ability of HG-induced HUVECs treated or transfected with exosomes or miRNA agomirs. The total numbers of branching points $(p<0.001)$ and total tube length $(p<0.0001)$ were increased in miR221-3p agomir-transfected HUVECs compared with NC agomir-transfected HUVECs (Fig. 7F, G). Moreover,

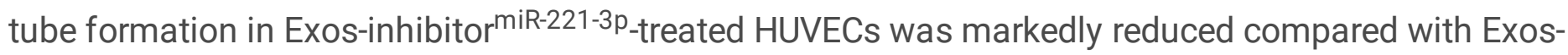
inhibitor ${ }^{N C}$-treated HUVECs. Thus, hucMSC-Exos could improve the function of endothelial cells by targeting the PTEN/AKT/HIF-1a/VEGF signaling pathways through miR-221-3p.

\section{MiR-221-3p in hucMSC-Exos exerts angiogenic effects on wound healing in diabetic mice}

Finally, we sought to evaluate the ability of miR-221-3p to facilitate wound healing of diabetic mice in vivo. Full-thickness wounds on the back were locally injected with NC agomirs, Exos-inhibitor ${ }^{N C}$, Exosinhibitor ${ }^{m i R-221-3 p}$, or miR-221-3p agomirs every other day post wounding. The wound healing process was significantly accelerated by local injection of Exos-inhibitor ${ }^{N C}$ and miR-221-3p agomirs compared with Exos-inhibitor ${ }^{m i R-221-3 p}$ and NC agomirs (Fig. 8A, B). Higher local MPU ratios were also observed in Exos-inhibitor ${ }^{N C}$ and miR-221-3p agomir-treated mice, reflecting enhanced blood flow at wound sites ( $p<$ 0.05) (Fig. 8C). Consistent with the wound healing and MPU ratio analyses, Exos-inhibitor ${ }^{N C}$ and miR-221$3 p$ agomirs significantly increased the numbers of newly formed blood vessels $(p<0.0001)$ (Fig. 8D, $E)$ and CD31 expression levels $(p<0.001)$ (Fig. 8F, G). Furthermore, Exos-inhibitor ${ }^{N C}$ induced improvements in angiogenesis that were diminished by treatment with Exos-inhibitormiR-221-3p (Fig. 8C-G). Collectively, these findings revealed that miR-221-3p plays a crucial role in hucMSC-Exos-dependent regulation of endothelial angiogenesis: suppression of miR-221-3p in hucMSC-Exos markedly decreased the regulatory effects of hucMSC-Exos, suggesting that these exosomes could represent novel therapeutic tools to promote diabetic wound healing.

\section{Discussion}

In the present study, we investigated the properties of hucMSC-Exos and their ability to enhance diabetic wound healing and angiogenesis. Potential underlying mechanisms were also explored including signaling in recipient cells and candidate miRNAs in hucMSC-Exos. Our results showed that hucMSCExos promoted diabetic wound healing and angiogenesis in vivo. In vitro, hucMSC-Exos facilitated proliferation, migration, and tube formation in HG-treated HUVECs, and mitigated the cellular senescence induced by HG. The AKT/HIF-1a/VEGF signaling pathways regulated by PTEN were activated by hucMSC-Exos in HG-treated HUVECs. Moreover, inhibition of PTEN mimicked the effects of hucMSC-Exos on endothelial cell function. MiR-221-3p was highly enriched in hucMSC-Exos. Using inhibitors and agomirs, we confirmed that miR-221-3p was a key player in the regulatory effects of hucMSC-Exos on diabetic wound healing and angiogenesis, and exerted its effects by targeting PTEN. 
Diabetic wounds are difficult to heal because of their complex underlying pathology. Significant attention has been paid to the treatment of diabetic wound [46]. Stem cells have been reported to have beneficial effects on diabetic wound repair [47]. However, because stem cell transplantation is limited by immune rejection, tumorigenicity, and ethical issues, this therapy is not widely available in the clinic. Recent studies have found that culture supernatants of stem cells can accelerate the repair of damaged tissue $[48,49]$. Accordingly, researchers are beginning to focus upon the exocrine function of stem cells. Exosomes are key paracrine factors of stem cells that can modulate the functions of target cells in wound healing. For treatment of diabetic wounds, MSC-derived exosomes are receiving more and more attention. Exosomes from bone marrow derived MSCs, especially those pretreated with atorvastatin, can be used to treat diabetic skin defects by improving the biological function of endothelial cells [22]. Recently, we reported that exosomes from human decidua-derived MSCs could enhance diabetic wound healing by optimizing functional states of fibroblasts [33]. In the current study, we showed the beneficial effects of hucMSC-Exos on diabetic wound healing. Such effects were not obvious in a previous study, perhaps because of the low concentrations of hucMSC-Exos used for treatment [26].

During wound healing, the formation of new blood vessels is crucial for delivering trophic factors to wound sites [50]. Under the hyperglycemic conditions of diabetes, glucose toxicity can lead to reduced cellular viability and increased senescence of endothelial cells [51], resulting in insufficient angiogenesis and low capillary density in diabetic wounds. Therefore, endothelial cells may be potential targets for ameliorating diabetic vascular complications. Using a rat skin burn model, Zhang et al. demonstrated that hucMSC-Exos promoted wound healing and angiogenesis in vivo via the Wnt4/ $\beta$-catenin pathway [52]. Under HG conditions, the functional states of endothelial cells differ from those under NG conditions, so whether hucMSC-Exos can perform a similar function needs to be confirmed. In the present study, we demonstrated that treatment with hucMSC-Exos boosted neovascularization in diabetic wounds. Using a suite of in vitro angiogenesis-related assays, we discovered that hucMSC-Exos could profoundly stimulate proliferation, migration and angiogenic tube formation of HG-treated HUVECs. These experiments confirmed the pro-angiogenic properties of hucMSC-Exos. Thus, the favorable effects of hucMSC-Exos on diabetic wound healing may be ascribed to their function in restoration of injured endothelial cells under HG conditions.

We next investigated the signaling pathways activated in HG-treated HUVECs by hucMSC-Exos. The AKT/HIF-1a/VEGF pathways have been demonstrated to augment several crucial processes associated with angiogenesis such as migration and tube formation of endothelial cells [53]. Previous studies have verified that PTEN is a negative regulator of the AKT/HIF-1a/VEGF pathways [54]. Thus, we examined whether downregulation of PTEN and subsequent activation of the AKT/HIF-1a/VEGF pathways were associated with hucMSC-Exos-mediated proangiogenesis. Our results showed that hucMSC-Exos inhibited the expression of PTEN and activated AKT/HIF-1a/VEGF signaling. Furthermore, we found that silencing PTEN expression could mimic the effects of hucMSC-Exos on endothelial angiogenesis. Hence, we concluded that the proangiogenic effects of hucMSC-Exos were mediated through the PTEN/AKT/HIF1a/VEGF pathways. 
MiRNAs are short RNA molecules that play vital roles in a wide array of biological processes [55]. MiRNAs are secreted into the extracellular space within exosomes, allowing for their transfer to recipient cells where they can mediate gene expression $[54,56]$. To understand the mechanisms through which hucMSC-Exos promoted angiogenesis, candidate miRNAs within hucMSC-Exos needed to be identified. Using miRNA microarrays and qRT-PCR, we found that miR-221-3p was highly enriched in hucMSC-Exos. MiR-221-3p has been identified in HUVECs and may play an important role in the regulation of angiogenesis. For example, notoginsenoside R1 increased miR-221-3p expression to inhibit apoptosis, inflammation, and oxidative stress in HUVECs treated with oxidized low-density lipoprotein [57]. By contrast, in the heart tissue of patients who experienced heart failure, overexpression of miR-221-3p inhibited proliferation, migration, and cord formation of endothelial cells by targeting HIF-1a [58]. Thus, the effects of miR-221-3p may vary in different tissues. Exosomal miR-221-3p was also reported to promote angiogenesis. In carcinoma tissue, exosomal miR-221-3p secreted by cancer cells enhances invasion, migration, and angiogenesis of microvascular endothelial cells [39, 43]. However, in diabetic wounds, the functions of miR-221-3p derived from hucMSC-Exos in angiogenesis remain unclear. In the current study, we detected high levels of miR-221-3p in hucMSC-Exos. We then incubated HUVECs with hucMSC-Exos and found that the expression of miR-221-3p was remarkably enhanced. Administration of a specific inhibitor targeting miR-221-3p reversed, but did not entirely abolish, the effects of hucMSC-Exos on angiogenesis in vitro and in vivo. Moreover, agomirs of miR-221-3p could partially simulate the effects of hucMSC-Exos. On the basis of our findings, we propose that miR-221-3p is one of the principal mediators of hucMSC-Exo-induced regulation of endothelial cell properties. However, other signaling regulator may also be associated with this process, and will require further investigation.

Our study also confirmed the connection between signals in recipient cells and mediators within exosomes. Previous studies have demonstrated that the effects of miR-221-3p were mediated via increasing Akt kinase activity and inhibiting PTEN $[59,60]$. In agreement with these findings, our results showed that the inhibitors of miR-221-3p attenuated enhanced expression of PTEN and p-Akt induced by hucMSC-Exos. In contrast, agomirs of miR-221-3p functioned as well as hucMSC-Exos to increase the expression of PTEN and p-Akt. Bioinformatic analyses supported the finding that PTEN is a direct target of miR-221-3p. We conclude that high levels of miR-221-3p in hucMSC-Exos are responsible for modulating angiogenesis in diabetic wounds by targeting PTEN and activating the AKT/HIF-1a/VEGF pathways in HG-injured endothelial cells.

\section{Conclusions}

In conclusion, our findings demonstrate that hucMSC-Exos can potently better cutaneous wound repair in diabetic mice and the determining mechanism could be their enhancement of pro-angiogenic properties of endothelial cells in the diabetic wound sites, as hucMSC-Exos can boost the proliferation, migration and the angiogenic activities of HG-pretreated HUVECs. These therapeutic effects are proved to be miR221-3p-dependent, for the inhibition of miR-221-3p can significantly attenuate the hucMSC-Exos-induced impacts and agmirR-221-3p can partly achieve hucMSC-Exos-like effects. Moreover, miR-221-3p exerts functions directly via manipulating PTEN/AKT/HIF-1a/VEGF signaling pathway. It will also be of interest 
to analyze whether hucMSC-Exos have positive effects on the function of other cell types in diabetic wounds. These findings proposed that hucMSC-Exos may be a novel therapeutic approach to facilitate angiogenesis in diabetic wounds.

\section{Abbreviations}

miRNA: microRNA; hucMSC-Exos: exosomes derived from human umbilical cord mesenchymal stem cells; HG: high glucose; HUVEC: human umbilical vascular endothelial cell; miRNAs: microRNAs; PTEN: phosphatase and tensin homolog; DFU: diabetic foot ulceration; MSC: mesenchymal stem cell; hucMSC: human umbilical cord mesenchymal stem cell; BM-MSC: bone marrow mesenchymal stem cell; DLL4: Delta-like 4; NTA: nanoparticle tracking analyzer; TEM: transmission electron microscope; TSG101: tumor susceptibility gene101; NIH: National Institutes of Health; H\&E: hematoxylin and eosin; LSCl: laser speckle contrast imaging; MPU: mean perfusion unit; NG: normal glucose; CCK-8: cell counting kit-8; PI: propidium iodide; ROS: reactive oxygen species; NC: negative control; HRP: horseradish peroxidase;

\section{Declarations}

\section{Acknowledgements}

Not applicable.

\section{Authors' contributions}

QW: design and performance of the in vivo and in vitro studies, result analysis and writing original manuscript draft; $\mathrm{YW}$ : in vivo transplantation and imaging studies; KM: preparation of the cells and in vitro studies; XW: exosome studies and imaging; QL: RNA interference; BL: blood perfusion evaluation; WH: cell function evaluation; $\mathrm{HL}, \mathrm{XF}$ and $\mathrm{CZ}$ : design, result analysis, manuscript writing and editing, and funding acquisition. All authors have read and approved the final manuscript draft.

\section{Funding}

This work was supported by the National Nature Science Foundation of China (81830064, 81721092, 81901971); the National Key Research Development Plan (2017YFC1103304); the CAMS Innovation Fund for Medical Sciences (CIFMS, 2019-I2M-5-059); the Military Medical Research and Development Projects (AWS17J005, 2019-126) and the Beijing Municipal Natural Science Foundation (7202197, 7194316).

\section{Availability of data and materials}

The datasets used and/or analyzed during the current study are available from the corresponding author on reasonable request. 


\section{Ethics approval and consent to participate}

All animal procedures were approved by the Animal Research Committee of PLA General Hospital and were performed in accordance with established guidelines.

\section{Consent for publication}

Not applicable.

\section{Competing Interests}

The authors have declared that no competing interest exists.

\section{References}

1. Patel S, Srivastava S, Singh MR, Singh D. Mechanistic insight into diabetic wounds: Pathogenesis, molecular targets and treatment strategies to pace wound healing. Biomed Pharmacother. 2019;112:108615.

2. Jeffcoate WJ, Harding KG. Diabetic foot ulcers. Lancet. 2003;361(9368):1545-51.

3. Kant V, Gopal A, Kumar D, Pathak NN, Ram M, Jangir BL, et al. Curcumin-induced angiogenesis hastens wound healing in diabetic rats. J Surg Res. 2015;193(2):978-88.

4. Bakker K, Apelqvist J, Lipsky BA, Van Netten JJ. The 2015 IWGDF guidance documents on prevention and management of foot problems in diabetes: development of an evidence-based global consensus. Diabetes Metab Res Rev. 2016;32 Suppl 1:2-6.

5. Galkowska H, Wojewodzka U, Olszewski WL. Chemokines, cytokines, and growth factors in keratinocytes and dermal endothelial cells in the margin of chronic diabetic foot ulcers. Wound Repair Regen. 2006;14(5):558-65.

6. Falanga V. Wound healing and its impairment in the diabetic foot. Lancet. 2005;366(9498):1736-43.

7. Wu YS, Chen SN. Extracted Triterpenes from Antrodia cinnamomea Reduce the Inflammation to Promote the Wound Healing via the STZ Inducing Hyperglycemia-Diabetes Mice Model. Front Pharmacol. 2016;7:154.

8. Samsonraj RM, Raghunath M, Nurcombe V, Hui JH, van Wijnen AJ, Cool SM. Concise Review: Multifaceted Characterization of Human Mesenchymal Stem Cells for Use in Regenerative Medicine. Stem Cells Transl Med. 2017;6(12):2173-85.

9. Kim SM, Kim YH, Jun YJ, Yoo G, Rhie JW. The effect of diabetes on the wound healing potential of adipose-tissue derived stem cells. Int Wound J. 2016;13 Suppl 1:33-41.

10. Kim EK, Li G, Lee TJ, Hong JP. The effect of human adipose-derived stem cells on healing of ischemic wounds in a diabetic nude mouse model. Plast Reconstr Surg. 2011;128(2):387-94. 
11. Chen Y, Zhao Y, Chen W, Xie L, Zhao ZA, Yang J, et al. MicroRNA-133 overexpression promotes the therapeutic efficacy of mesenchymal stem cells on acute myocardial infarction. Stem Cell Res Ther. 2017;8(1):268.

12. Zhao J, Li X, Hu J, Chen F, Qiao S, Sun X, et al. Mesenchymal stromal cell-derived exosomes attenuate myocardial ischaemia-reperfusion injury through miR-182-regulated macrophage polarization. Cardiovasc Res. 2019;115(7):1205-16.

13. Shiota Y, Nagai A, Sheikh AM, Mitaki S, Mishima S, Yano S, et al. Transplantation of a bone marrow mesenchymal stem cell line increases neuronal progenitor cell migration in a cerebral ischemia animal model. Sci Rep. 2018;8(1):14951.

14. Hawkins KE, Corcelli M, Dowding K, Ranzoni AM, Vlahova F, Hau KL, et al. Embryonic Stem CellDerived Mesenchymal Stem Cells (MSCs) Have a Superior Neuroprotective Capacity Over Fetal MSCs in the Hypoxic-Ischemic Mouse Brain. Stem Cells Transl Med. 2018;7(5):439-49.

15. Wang Z, Zheng L, Lian C, Qi Y, Li W, Wang S. Human Umbilical Cord-Derived Mesenchymal Stem Cells Relieve Hind Limb Ischemia by Promoting Angiogenesis in Mice. Stem Cells Dev. 2019;28(20):1384-97.

16. Shen L, Zeng W, Wu YX, Hou CL, Chen W, Yang MC, et al. Neurotrophin-3 accelerates wound healing in diabetic mice by promoting a paracrine response in mesenchymal stem cells. Cell Transplant. 2013;22(6):1011-21.

17. Wang S, Yang H, Tang Z, Long G, Huang W. Wound Dressing Model of Human Umbilical Cord Mesenchymal Stem Cells-Alginates Complex Promotes Skin Wound Healing by Paracrine Signaling. Stem Cells Int. 2016;2016:3269267.

18. Jung JW, Kwon M, Choi JC, Shin JW, Park IW, Choi BW, et al. Familial occurrence of pulmonary embolism after intravenous, adipose tissue-derived stem cell therapy. Yonsei Med J. 2013;54(5):1293-6.

19. Barkholt L, Flory E, Jekerle V, Lucas-Samuel S, Ahnert P, Bisset L, et al. Risk of tumorigenicity in mesenchymal stromal cell-based therapies-bridging scientific observations and regulatory viewpoints. Cytotherapy. 2013;15(7):753-9.

20. Furlani D, Ugurlucan M, Ong L, Bieback K, Pittermann E, Westien I, et al. Is the intravascular administration of mesenchymal stem cells safe? Mesenchymal stem cells and intravital microscopy. Microvasc Res. 2009;77(3):370-6.

21. Lamichhane TN, Sokic S, Schardt JS, Raiker RS, Lin JW, Jay SM. Emerging roles for extracellular vesicles in tissue engineering and regenerative medicine. Tissue Eng Part B Rev. 2015;21(1):45-54.

22. Yu M, Liu W, Li J, Lu J, Lu H, Jia W, et al. Exosomes derived from atorvastatin-pretreated MSC accelerate diabetic wound repair by enhancing angiogenesis via AKT/eNOS pathway. Stem Cell Res Ther. 2020;11(1):350.

23. Ti D, Hao H, Tong C, Liu J, Dong L, Zheng J, et al. LPS-preconditioned mesenchymal stromal cells modify macrophage polarization for resolution of chronic inflammation via exosome-shuttled let-7b. J Transl Med. 2015;13:308. 
24. Liang X, Zhang L, Wang S, Han Q, Zhao RC. Exosomes secreted by mesenchymal stem cells promote endothelial cell angiogenesis by transferring miR-125a. J Cell Sci. 2016;129(11):2182-9.

25. Xiong Y, Chen L, Yan C, Zhou W, Endo Y, Liu J, et al. Circulating Exosomal miR-20b-5p Inhibition Restores Wnt9b Signaling and Reverses Diabetes-Associated Impaired Wound Healing. Small. 2020;16(3):e1904044.

26. Yang J, Chen Z, Pan D, Li H, Shen J. Umbilical Cord-Derived Mesenchymal Stem Cell-Derived Exosomes Combined Pluronic F127 Hydrogel Promote Chronic Diabetic Wound Healing and Complete Skin Regeneration. Int J Nanomedicine. 2020;15:5911-26.

27. Jia G, Han Y, An Y, Ding Y, He C, Wang X, et al. NRP-1 targeted and cargo-loaded exosomes facilitate simultaneous imaging and therapy of glioma in vitro and in vivo. Biomaterials. 2018;178:302-16.

28. Hu Y, Rao SS, Wang ZX, Cao J, Tan YJ, Luo J, et al. Exosomes from human umbilical cord blood accelerate cutaneous wound healing through miR-21-3p-mediated promotion of angiogenesis and fibroblast function. Theranostics. 2018;8(1):169-84.

29. Baptista VIA, Quintana HT, Lazzarin MC, Benfato ID, De Carvalho FP, Le Sueur-Maluf L, et al. Short time insulin treatment post burn improves elastic-collagen rearrangement and reepithelization. Connect Tissue Res. 2019;60(3):230-9.

30. Liu S, Wu F, Gu S, Wu T, Chen S, Chen S, et al. Gene Silencing via PDA/ERK2-siRNA-Mediated Electrospun Fibers for Peritendinous Antiadhesion. Adv Sci (Weinh). 2019;6(2):1801217.

31. Kupcova Skalnikova H. Proteomic techniques for characterisation of mesenchymal stem cell secretome. Biochimie. 2013;95(12):2196-211.

32. Wang A, Liu J, Zhuang X, Yu S, Zhu S, Liu Y, et al. Identification and Comparison of piRNA Expression Profiles of Exosomes Derived from Human Stem Cells from the Apical Papilla and Bone Marrow Mesenchymal Stem Cells. Stem Cells Dev. 2020;29(8):511-20.

33. Bian X, Li B, Yang J, Ma K, Sun M, Zhang C, et al. Regenerative and protective effects of dMSC-sEVs on high-glucose-induced senescent fibroblasts by suppressing RAGE pathway and activating Smad pathway. Stem Cell Res Ther. 2020;11(1):166.

34. Wang X, Ackermann M, Neufurth M, Wang S, Li Q, Feng Q, et al. Restoration of Impaired Metabolic Energy Balance (ATP Pool) and Tube Formation Potential of Endothelial Cells under "high glucose", Diabetic Conditions by the Bioinorganic Polymer Polyphosphate. Polymers (Basel). 2017;9(11).

35. Park JH, Lee JY, Shin DH, Jang KS, Kim HJ, Kong G. Loss of Mel-18 induces tumor angiogenesis through enhancing the activity and expression of HIF-1a mediated by the PTEN/PI3K/Akt pathway. Oncogene. 2011;30(45):4578-89.

36. Worby CA, Dixon JE. PTEN. Annu Rev Biochem. 2014;83:641-69.

37. Xiang T, Lin YX, Ma W, Zhang HJ, Chen KM, He GP, et al. Vasculogenic mimicry formation in EBVassociated epithelial malignancies. Nat Commun. 2018;9(1):5009.

38. Tkach M, Théry C. Communication by Extracellular Vesicles: Where We Are and Where We Need to Go. Cell. 2016;164(6):1226-32. 
39. Wu XG, Zhou CF, Zhang YM, Yan RM, Wei WF, Chen XJ, et al. Cancer-derived exosomal miR-221-3p promotes angiogenesis by targeting THBS2 in cervical squamous cell carcinoma. Angiogenesis. 2019;22(3):397-410.

40. Xu CH, Liu Y, Xiao LM, Chen LK, Zheng SY, Zeng EM, et al. Silencing microRNA-221/222 cluster suppresses glioblastoma angiogenesis by suppressor of cytokine signaling-3-dependent JAK/STAT pathway. J Cell Physiol. 2019;234(12):22272-84.

41. Yang F, Wang W, Zhou C, Xi W, Yuan L, Chen X, et al. MiR-221/222 promote human glioma cell invasion and angiogenesis by targeting TIMP2. Tumour Biol. 2015;36(5):3763-73.

42. Chen WX, Hu Q, Qiu MT, Zhong SL, Xu JJ, Tang JH, et al. miR-221/222: promising biomarkers for breast cancer. Tumour Biol. 2013;34(3):1361-70.

43. Zhang L, Li H, Yuan M, Li M, Zhang S. Cervical Cancer Cells-Secreted Exosomal microRNA-221-3p Promotes Invasion, Migration and Angiogenesis of Microvascular Endothelial Cells in Cervical Cancer by Down-Regulating MAPK10 Expression. Cancer Manag Res. 2019;11:10307-19.

44. Lightell DJ, Jr., Moss SC, Woods TC. Upregulation of miR-221 and -222 in response to increased extracellular signal-regulated kinases $1 / 2$ activity exacerbates neointimal hyperplasia in diabetes mellitus. Atherosclerosis. 2018;269:71-8.

45. Du W, Zhang K, Zhang S, Wang R, Nie Y, Tao H, et al. Enhanced proangiogenic potential of mesenchymal stem cell-derived exosomes stimulated by a nitric oxide releasing polymer. Biomaterials. 2017;133:70-81.

46. Berlanga-Acosta J, Armstrong D, Schultz G, Herrera-Martinez L. Chronic wounds with emphasis in diabetic foot ulcers. Biomed Res Int. 2014;2014:890352.

47. Maranda EL, Rodriguez-Menocal L, Badiavas EV. Role of Mesenchymal Stem Cells in Dermal Repair in Burns and Diabetic Wounds. Curr Stem Cell Res Ther. 2017;12(1):61-70.

48. Fouraschen SM, Pan Q, de Ruiter PE, Farid WR, Kazemier G, Kwekkeboom J, et al. Secreted factors of human liver-derived mesenchymal stem cells promote liver regeneration early after partial hepatectomy. Stem Cells Dev. 2012;21(13):2410-9.

49. Ionescu L, Byrne RN, van Haaften T, Vadivel A, Alphonse RS, Rey-Parra GJ, et al. Stem cell conditioned medium improves acute lung injury in mice: in vivo evidence for stem cell paracrine action. Am J Physiol Lung Cell Mol Physiol. 2012;303(11):L967-77.

50. Casado-Díaz A, Quesada-Gómez JM, Dorado G. Extracellular Vesicles Derived From Mesenchymal Stem Cells (MSC) in Regenerative Medicine: Applications in Skin Wound Healing. Front Bioeng Biotechnol. 2020;8:146.

51. Rezabakhsh A, Nabat E, Yousefi M, Montazersaheb S, Cheraghi O, Mehdizadeh A, et al. Endothelial cells' biophysical, biochemical, and chromosomal aberrancies in high-glucose condition within the diabetic range. Cell Biochem Funct. 2017;35(2):83-97.

52. Zhang $B$, Wu $X$, Zhang $X$, Sun $Y$, Yan $Y$, Shi $H$, et al. Human umbilical cord mesenchymal stem cell exosomes enhance angiogenesis through the Wnt4/ $\beta$-catenin pathway. Stem Cells Transl Med. 2015;4(5):513-22. 
53. Zhang Y, Liu NM, Wang Y, Youn JY, Cai H. Endothelial cell calpain as a critical modulator of angiogenesis. Biochim Biophys Acta Mol Basis Dis. 2017;1863(6):1326-35.

54. Pulido R. PTEN Inhibition in Human Disease Therapy. Molecules. 2018;23(2).

55. Rupaimoole R, Slack FJ. MicroRNA therapeutics: towards a new era for the management of cancer and other diseases. Nat Rev Drug Discov. 2017;16(3):203-22.

56. Chen Y, Pfeifer A. Brown Fat-Derived Exosomes: Small Vesicles with Big Impact. Cell Metab. 2017;25(4):759-60.

57. Zhu L, Gong X, Gong J, Xuan Y, Fu T, Ni S, et al. Notoginsenoside R1 upregulates miR-221-3p expression to alleviate ox-LDL-induced apoptosis, inflammation, and oxidative stress by inhibiting the TLR4/NF-kB pathway in HUVECs. Braz J Med Biol Res. 2020;53(6):e9346.

58. Li Y, Yan C, Fan J, Hou Z, Han Y. MiR-221-3p targets Hif-1a to inhibit angiogenesis in heart failure. Lab Invest. 2021;101(1):104-15.

59. Sun L, Zhu W, Zhao P, Zhang J, Lu Y, Zhu Y, et al. Down-Regulated Exosomal MicroRNA-221 - 3p Derived From Senescent Mesenchymal Stem Cells Impairs Heart Repair. Front Cell Dev Biol. 2020;8:263.

60. Kim YC, Cutler ML. MicroRNA-Dependent Targeting of RSU1 and the IPP Adhesion Complex Regulates the PTEN/PI3K/AKT Signaling Pathway in Breast Cancer Cell Lines. Int J Mol Sci. 2020;21(15).

\section{Figures}


A



B



$\mathrm{C}_{75 \mathrm{kDa}}$

$60 \mathrm{kDa}$

$24 \mathrm{kDa}-\mathrm{CD} 9$

$23 \mathrm{kDa}-\mathrm{CD81}$

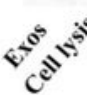

D
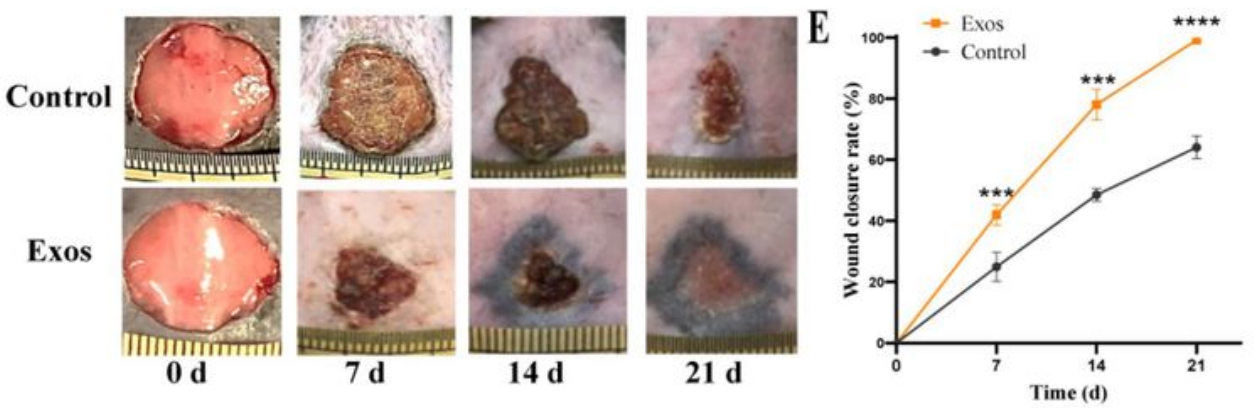

F

Control

$7 \mathrm{~d}$

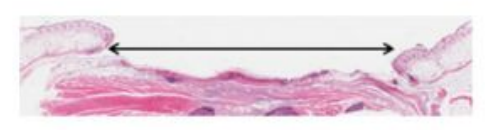

Exos



$14 \mathrm{~d}$
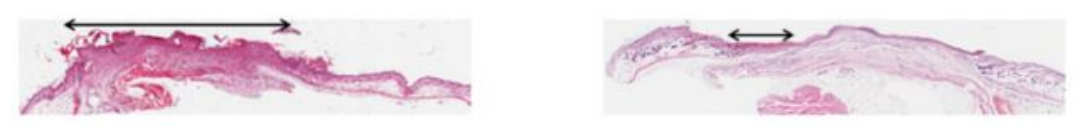

$21 \mathrm{~d}$

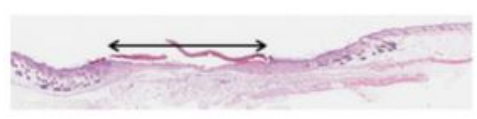

G

Control

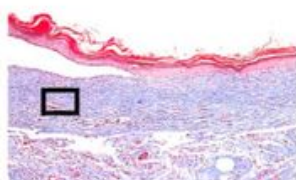

$100 \times$

$400 \times$

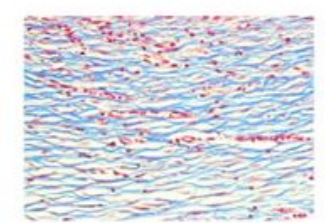

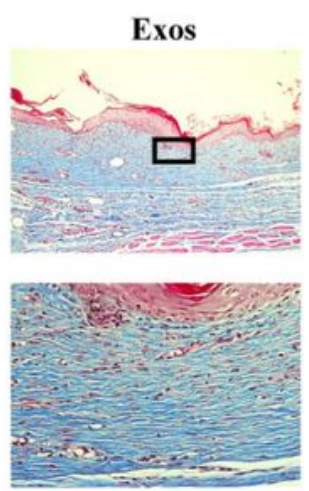

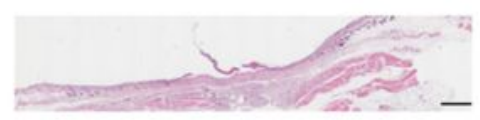

H



\section{Figure 1}

Characteristics of hucMSC-Exos and improvement of diabetic wound healing by hucMSC-Exos. (A) Particle size distribution and concentration results of hucMSC-Exos measured by qNano analysis. (B) TEM photomicrographs of hucMSC-Exos. Scale bar: $50 \mathrm{~nm}$. (C) Western blot of exosomal markers in hucMSC-Exos. (D) Gross view of wounds treated with PBS or hucMSC-Exos at planed days post wounding. (E) The rates of wound closure among groups receiving different treatments were evaluated 
and quantified by using ImageJ software ( $n=5$ for each group). ${ }^{\star \star \star} p<0.001, * \star \star \star p<0.0001$. (F) H\&E staining of wound sections treated with PBS or hucMSC-Exos at planed days after operation. The black arrows demonstrate the edges of the scar. Scale bar: $500 \mu \mathrm{m}$. (G) Masson's trichrome stained wound sections treated with PBS or hucMSC-Exos. (H) Quantification of collagen volume fraction (CVF) from PBS or hucMSC-Exos treated groups. ${ }^{* \star} p<0.001$.

A

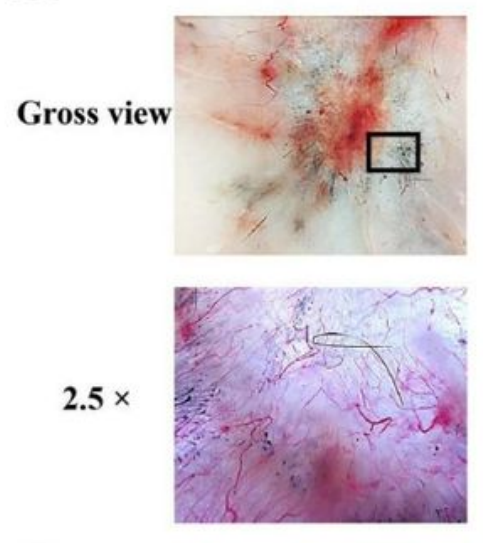

C



D



$\mathbf{E}$



Exos


Exos



B
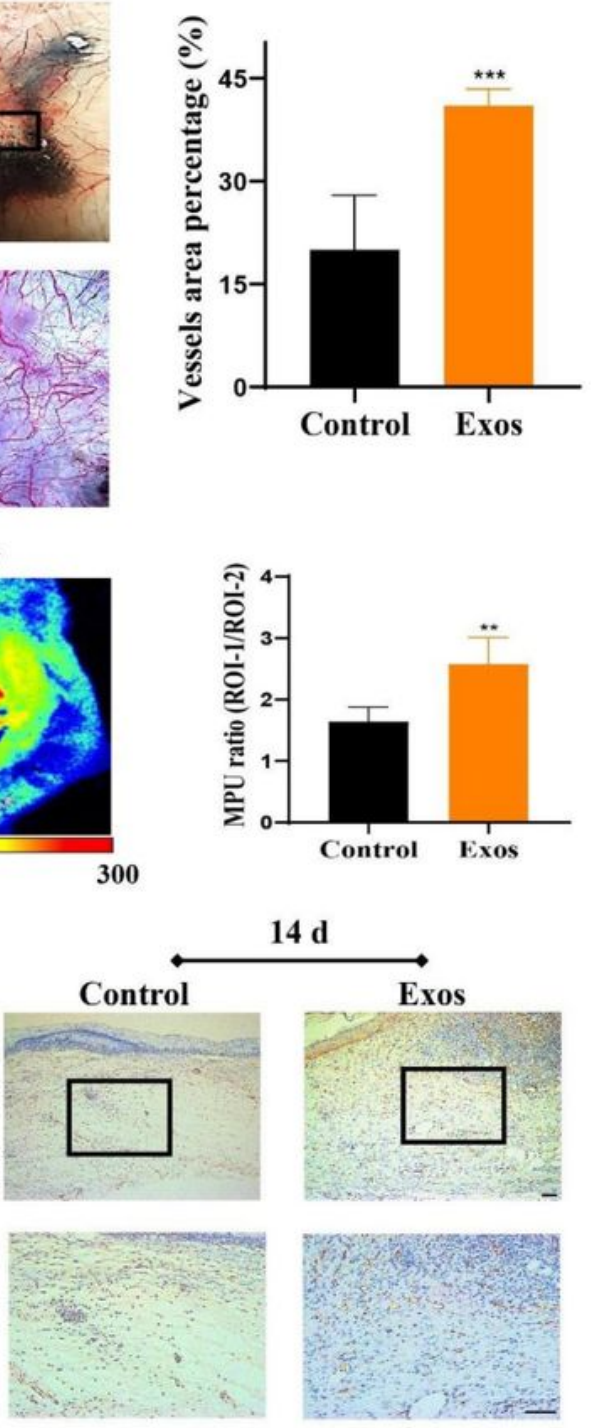

14 d

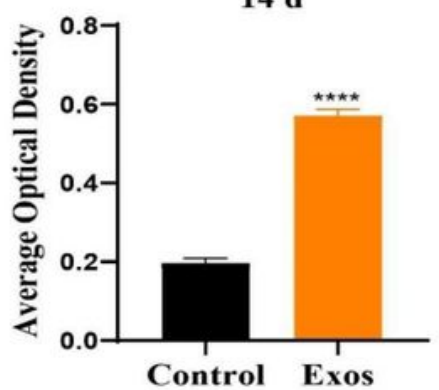

Figure 2 
HucMSC-Exos promote angiogenesis in the wound sites of diabetic mice. (A) Gross view of wounds that received different treatments at day 21 post wounding. Newly-formed blood vessels were found in the wound sections. (B) Quantitative analysis of vessel area in the control and hucMSC-Exos treated groups ( $n=5$ for per group). ${ }^{* \star *} p<0.001$. (C) The blood flows at the wound sections of different groups were evaluated by doppler of animals, flux images of the wounds were analyzed to detect the MPU ratio which was counted by comparing the MPU of the wounding area (ROI-1) with the area around the wounding section (ROI-2) ( $n=5$ for per group). ${ }^{*} p<0.01$. (D) CD31 staining of wound sites in the control and hucMSC-Exos treated groups. Scale bar: $200 \mu \mathrm{m}$. (E) Quantitation of average optical density (AOD) of




A

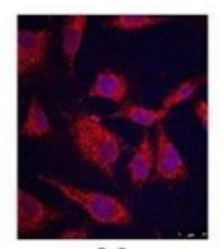

0 h



$1.5 \mathrm{~h}$



3 h


C


E

$\mathbf{0} \mathbf{h}$
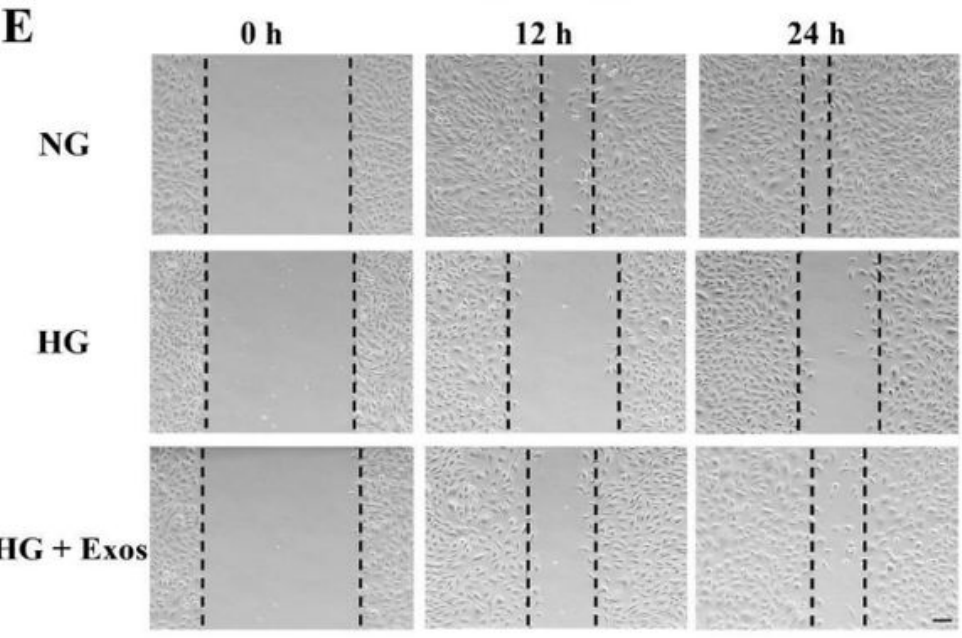

F

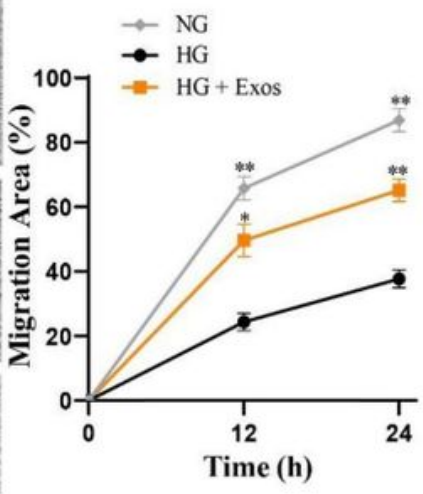

G

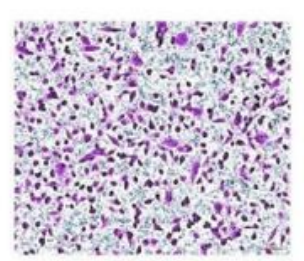

NG

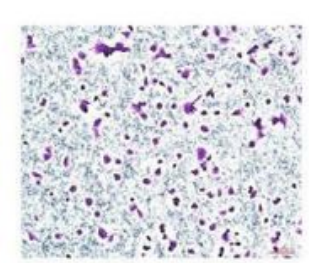

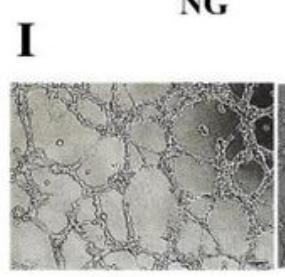

NG
HG

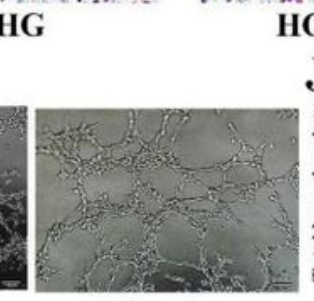

HG + Exos

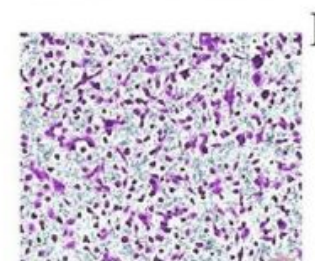

HG + Exos
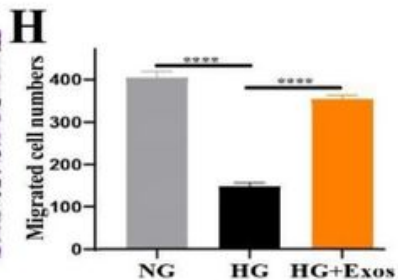
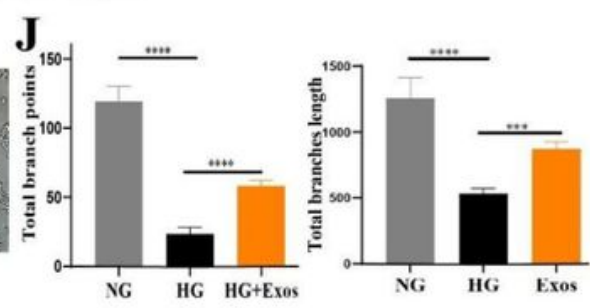



HG

\section{Figure 3}

HucMSC-Exos improve the function of HG-treated HUVECs in vitro. (A) Laser confocal images of HUVECs that were co-cultured with PKH67-labeled hucMSC-Exos (green). CD31 antibody (red) and DPAI (blue)were used to marked the cells. The observation timing is indicated below the images. Scale Bar: $25 \mu \mathrm{m}$. (B) CCK-8 analysis was conducted to estimate the proliferation of HUVEC incubated with NG, HG and HG + hucMSC-Exos. ${ }^{*} p<0.05,{ }^{* *} p<0.01,{ }^{* * * *} p<0.0001$. (C-D) Effects of hucMSC-Exos on the cell cycle 



assay and transwell assay were performed to assess the migration ability of HUVECs from different groups, as well as quantitative analysis. Scale Bar: $100 \mu \mathrm{m}$. ${ }^{\star} \mathrm{p}<0.05,{ }^{*} \mathrm{p}<0.01,{ }^{* \star *} \mathrm{p}<0.0001$. (I) The images of tube formation of HUVECs with different treatments. Scale Bar: $100 \mu \mathrm{m}$. (J) Quantitative analysis of tube formation ability. ${ }^{\star * \star} p<0.001, * \star \star * p<0.0001$.

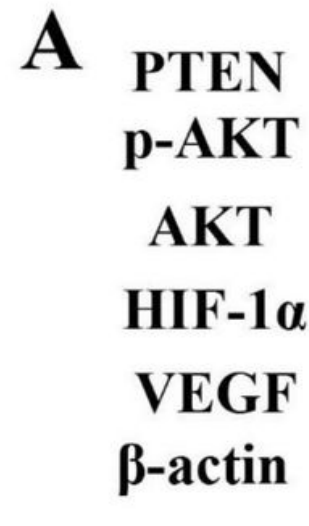

C

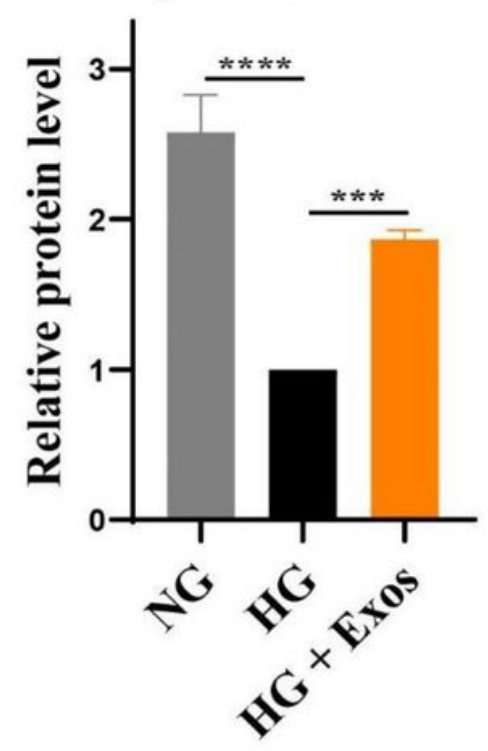

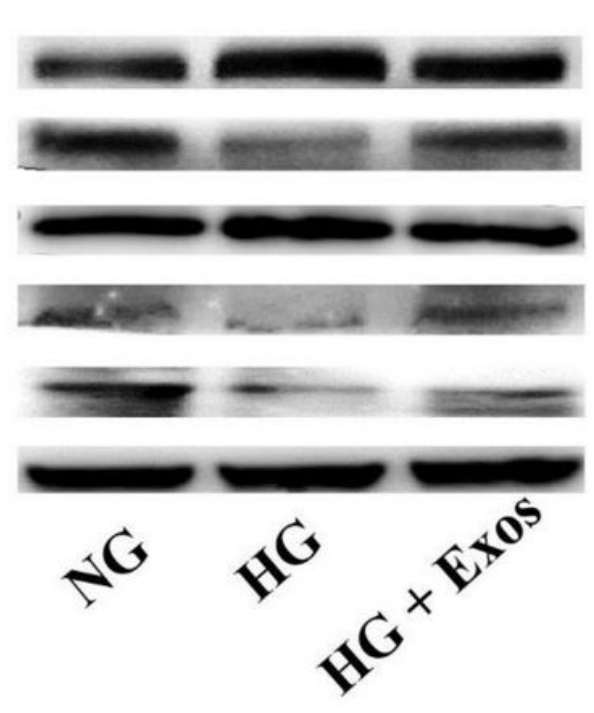


B
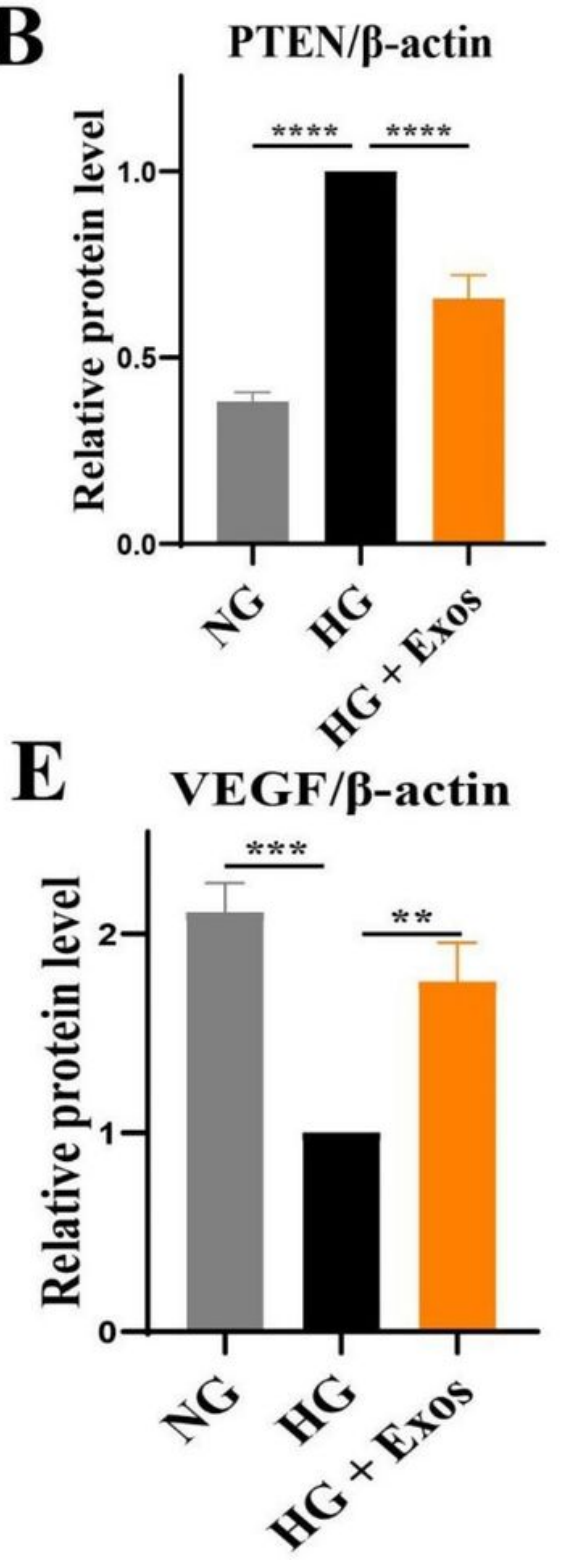

Figure 4

HucMSC-Exos regulate PTEN/AKT/HIF-1a/VEGF signaling pathway in HG-treated HUVECs. (A) Representative photographs of the PTEN, p-AKT, HIF-1a, and VEGF expression in HUVECs detected by Western blot. (B) PTEN quantitative bar graph of western blots. The results are normalized to $\beta$-actin expression. ${ }^{* \star \star} p<0.0001$. (C) $p$-AKT quantitative bar graph of western blots. The results are normalized to AKT expression. ${ }^{\star \star \star} p<0.001,{ }^{\star \star \star \star} p<0.0001$. (D) HIF-1 a quantitative bar graph of western blots. The 
results are normalized to $\beta$-actin expression. ${ }^{\star \star} p<0.01,{ }^{\star \star \star} p<0.001$. (E) VEGF quantitative bar graph of western blots. The results are normalized to $\beta$-actin expression. ${ }^{\star \star} p<0.01,{ }^{\star \star \star} p<0.001$.
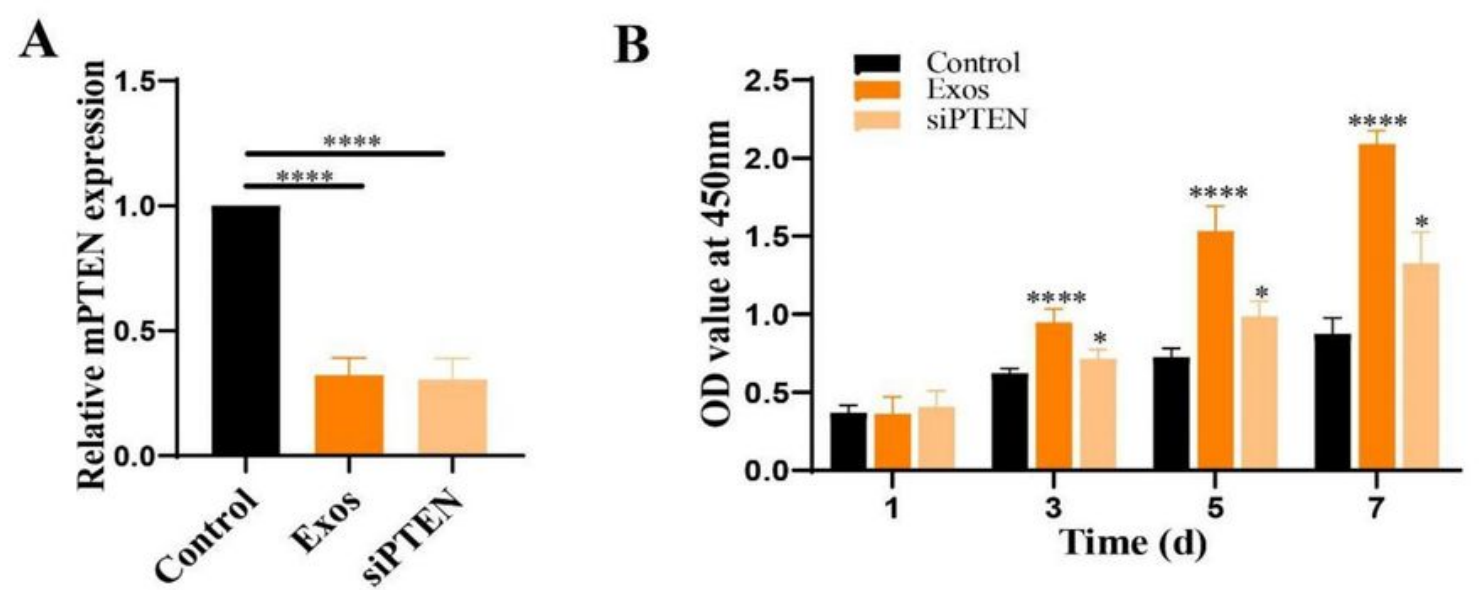

C
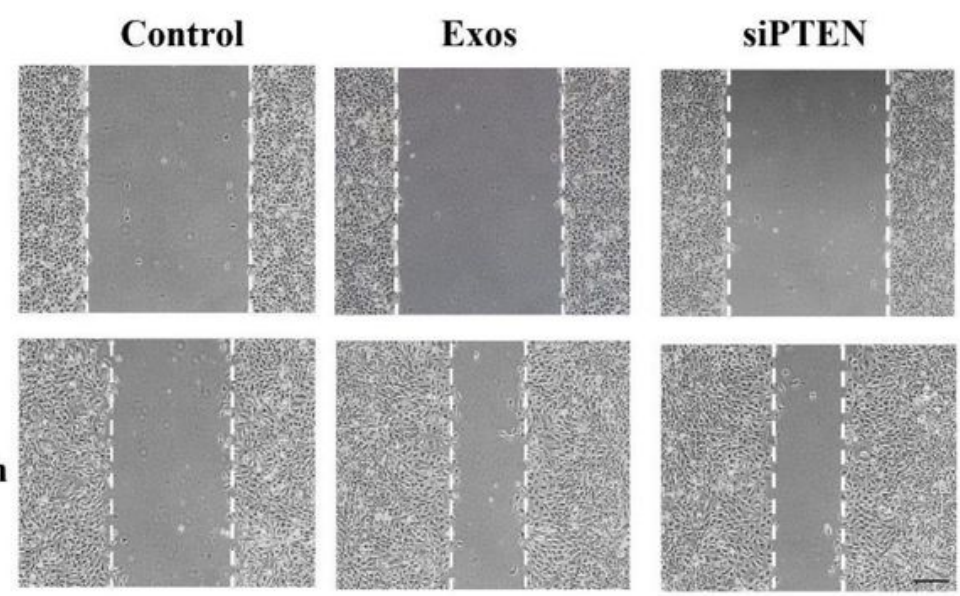

D

$\mathbf{0}$ h

$12 \mathrm{~h}$
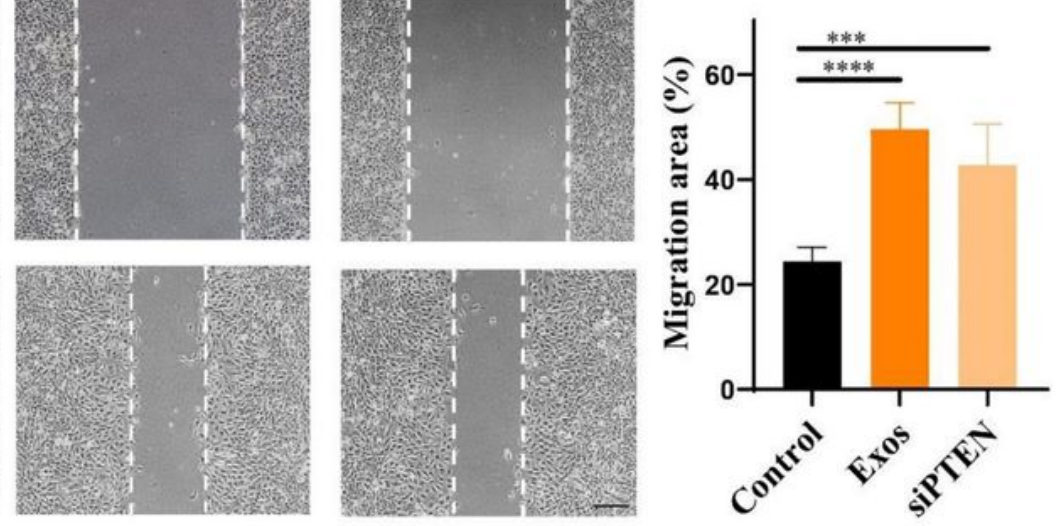

$\mathbf{E}$
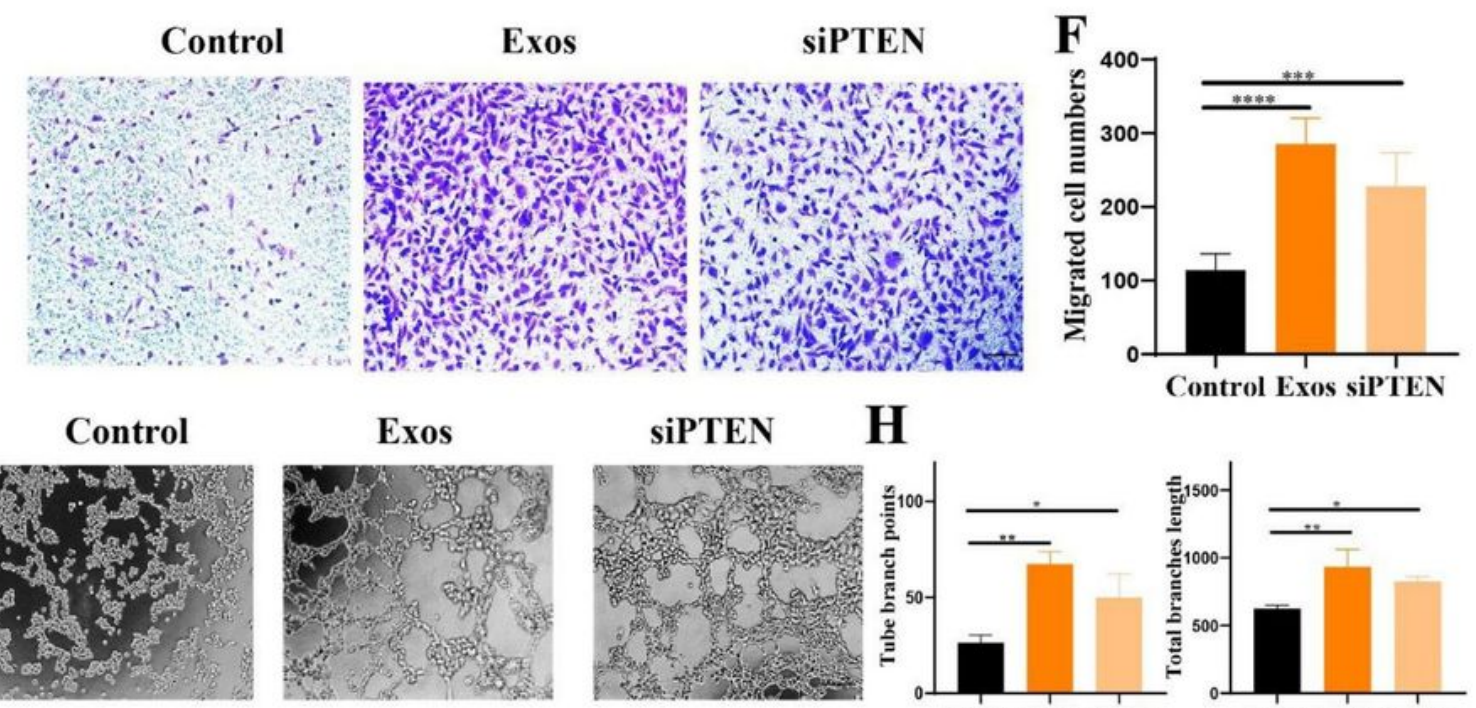

G



SiPTEN

H


Figure 5

PTEN knockdown generates hucMSC-Exos-like beneficial effects on HG-treated HUVECs. (A) The expression level of mPTEN of HUVECs from control, hucMSC-Exos and siPTEN groups ( $n=5$ per group). $\star \star \star * p<0.0001$. (B) Proliferation rates of HUVECs from different groups were measured by CCK-8 assay. 
${ }^{\star} p<0.05,{ }^{\star * \star \star} p<0.0001$. (C-F) Migration ability of HUVECs with different treatments are measured by wound scratch and transwell migration assays ( $n=5$ per group). Scale Bar: $100 \mu \mathrm{m} .{ }^{\star \star \star} p<0.001$, ${ }^{\star \star \star \star} p<$ 0.0001. (G) Effects of different treatments on the tube formation ability of HUVECs ( $n=5$ per group).

Scale Bar: $100 \mu \mathrm{m}$. $(\mathrm{H})$ Total branch points and tube length were quantified using ImageJ software. ${ }^{*} \mathrm{p}<$ $0.05, * * p<0.01$.

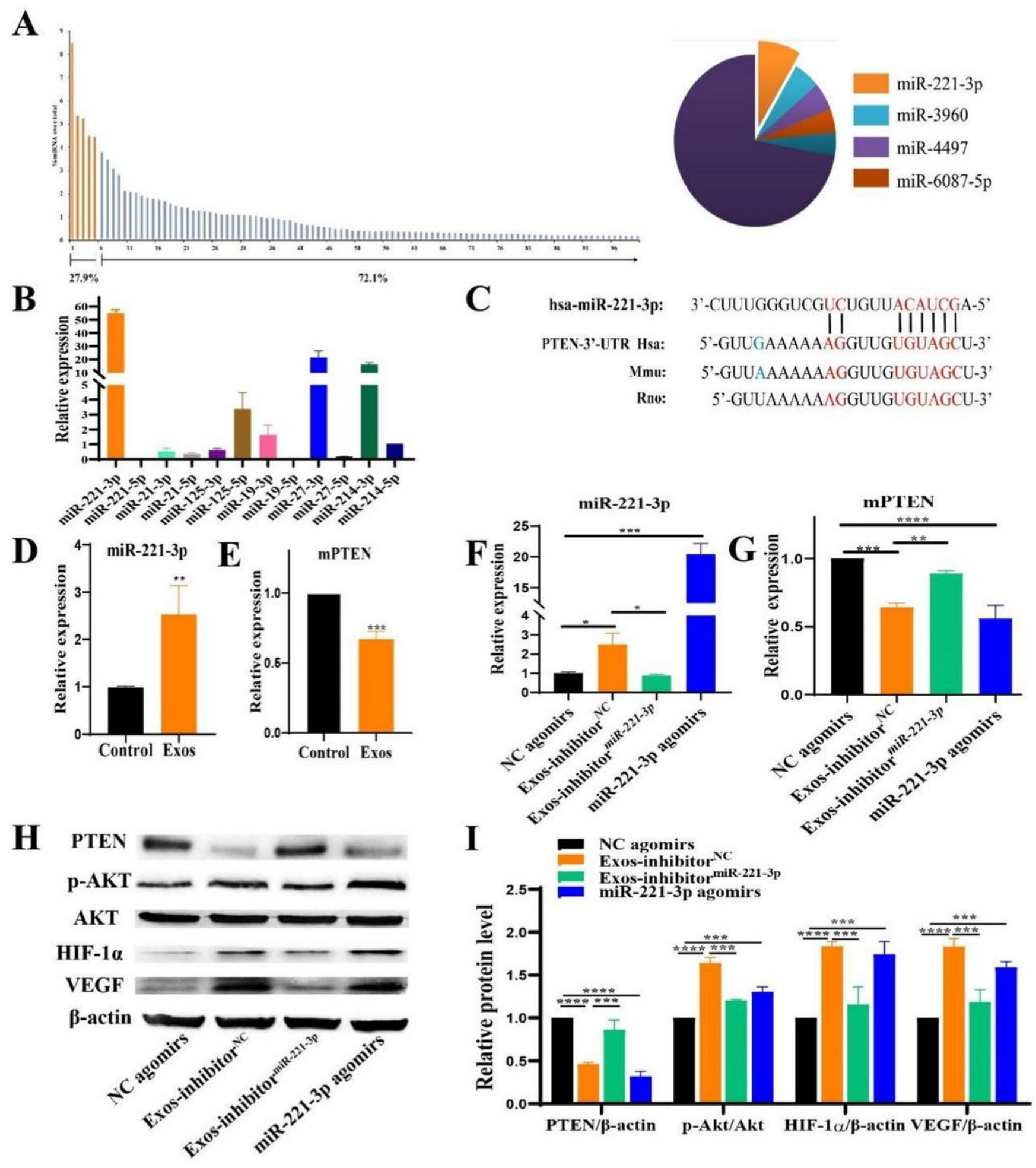

Figure 6 
MiR-221-3p in hucMSC-Exos downregulates PTEN and activates AKT/HIF-1a/VEGF pathway in HGtreated HUVECs. (A) Proportion of miRNAs in total miRNA reads. (B) Detection of the expression of the indicated miRNAs by qRT-PCR analysis ( $\mathrm{n}=3$ per group). (C) Putative miR-221-3p binding sites(red) in the $3^{\prime}$ - UTRs of PTEN from indicated species. (D) HUVECs incubated with hucMSC-Exos for $4.5 \mathrm{~h}$ showed higher expression levels of miR-221-3p than control did ( $n=3$ per group). ${ }^{\star} p<0.01$. (E) HUVECs incubated with hucMSC-Exos for $24 \mathrm{~h}$ showed lower level of mPTEN ( $n=3$ per group). ${ }^{\star \star \star} p<0.001$. (F) The miR-221-3p expression level in HUVECs treated with NC agomirs, Exos-inhibitorNC, Exos-inhibitor miR-221-3p, and miR-221-3p agomirs. ${ }^{\star} p<0.05,{ }^{* \star *} p<0.001$. (G) Expression of mPTEN in HUVECs after incubation with NC agomirs, Exos-inhibitorNC, Exos-inhibitor miR-221-3p, and miR-221-3p agomirs for 24 $\mathrm{h}\left(\mathrm{n}=3\right.$ per group). ${ }^{\star \star} \mathrm{p}<0.01,{ }^{* \star} \mathrm{p}<0.001,{ }^{* \star \star *} \mathrm{p}<0.0001$. $(\mathrm{H}-\mathrm{I})$ The expression level of PTEN, $\mathrm{p}-\mathrm{AKT}, \mathrm{HIF-}$ 1 , and VEGF and the quantification results. The results of PTEN, HIF-1, and VEGF are normalized to $\beta$ actin expression and the result of $p$-AKT is normalized to AKT expression ( $n=3$ per group). ${ }^{* \star} p<0.001$, $\star \star \star \star p<0.0001$. 



D

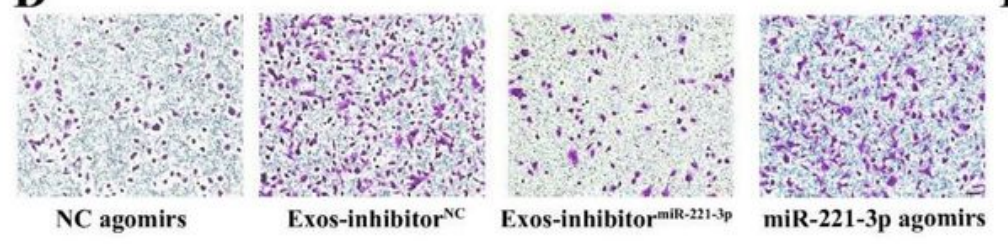

$\mathbf{E}$

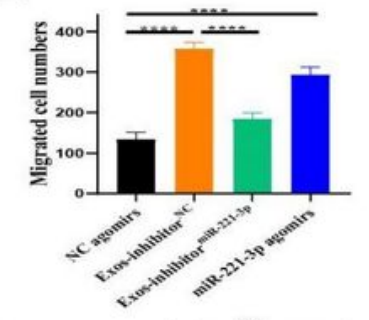

F

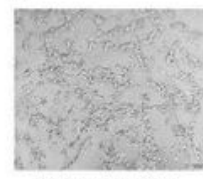

NC agomirs

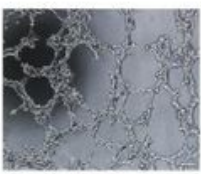

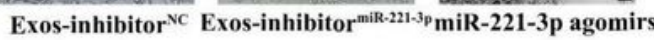

G
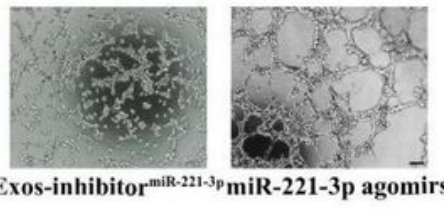

\section{Figure 7}

MiR-221-3p in hucMSC-Exos promotes proliferation, migration and tube formation of HG-treated HUVECs. (A) Proliferation rates of HUVECs from NC agomirs, Exos-inhibitorNC, Exos-inhibitor miR-221-3p, and miR-

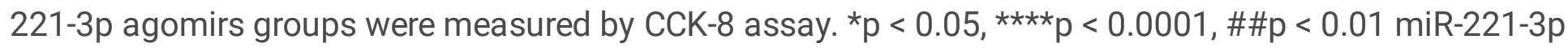
agomirs vs NC agomirs, \#\#\#p < 0.001 miR-221-3p agomirs vs NC agomirs. (B-C) Migration ability of HGinduced HUVECs with different treatments was measured by wound scratch assay ( $n=5$ per group). 
Scale Bar: $100 \mu \mathrm{m} .{ }^{\star \star \star *} \mathrm{p}<0.0001$ Exos-inhibitor miR-221-3p vs Exos-inhibitorNC, \#\#\#p < $0.001 \mathrm{miR}-221-$ $3 p$ agomirs vs NC agomirs. (D-E) Migration ability of HG-induced HUVECs measured by transwell migration assay ( $n=5$ per group). Scale Bar: $100 \mu$ m. ${ }^{* \star \star *} p<0.0001$. (F) Effects of different treatments on the tube formation ability of HG-induced HUVECs. Scale Bar: $100 \mu \mathrm{m}$. (G) Total branch points and tube length were quantified using ImageJ software ( $n=5$ per group). ${ }^{* \star} p<0.001, * \star \star \star p<0.0001$.

A NC-agomirs

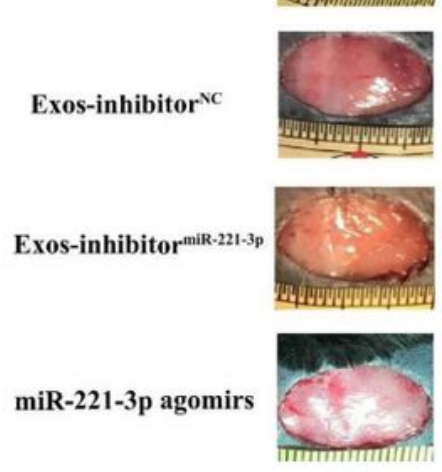

C

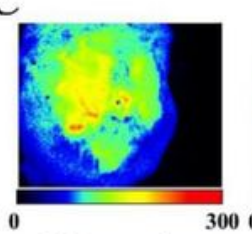

NC agomirs

D

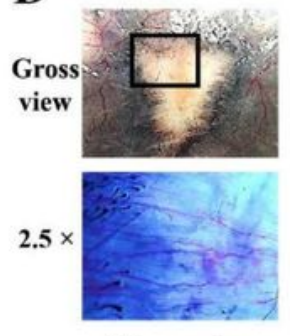

NC agomirs
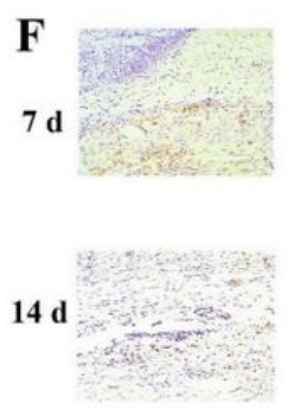

NC agomirs


7 d


$14 \mathrm{~d}$


21 d
B


$\mathbf{E}$



Exos-inhibitor $^{\text {NC }}$ Exos-inhibitor ${ }^{\text {miR-221-3p }}$ miR-221-3p agomirs
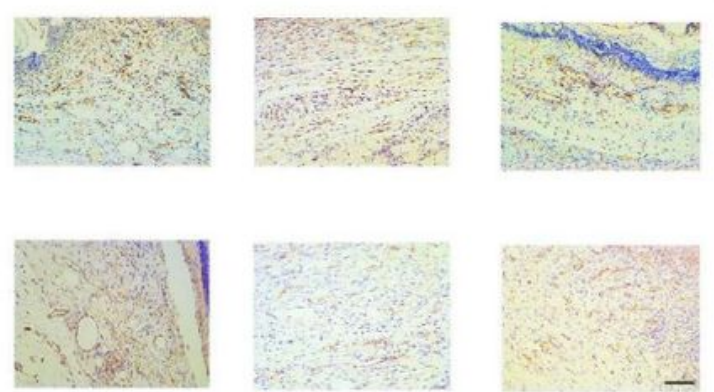


miR-221-3p agomirs



Figure 8 
MiR-221-3p in hucMSC-Exos exerts angiogenic effects on wound healing in diabetic mice. (A) Gross view of wounds from NC agomirs, Exos-inhibitorNC, Exos-inhibitor miR-221-3p, and miR-221-3p agomirs groups at day 0, 7, 14 and 21 post wounding. (B) The rates of wound closure among groups receiving different treatments were evaluated and quantified by using Image J software ( $\mathrm{n}=5$ per group). ${ }^{\star \star \star} \mathrm{p}<$ 0.001 Exos-inhibitor miR-221-3p vs Exos-inhibitorNC, ${ }^{* \star *} p<<0.0001$ Exos-inhibitor miR-221-3p vs ExosinhibitorNC, \#\#p < 0.01 miR-221-3p agomirs vs NC agomirs, \#\#\#p < 0.001 miR-221-3p agomirs vs NC agomirs. (C) The blood flows at the wound sections of different groups were evaluated by doppler of animals. Flux images of wounds were analyzed to detect the MPU ratio which was counted by comparing the MPU of the wounding area (ROI- 1 ) with the area around the wounding section (ROI-2) $(n=5$ per group). ${ }^{*} p<0.05,{ }^{*} p<0.01$. (D) Gross view of wounds that received different treatments at day 21 post wounding. Newly-formed blood vessels were found in the wound sections. (E) Quantitative analysis of vessel area in four groups ( $n=5$ per group). ${ }^{\star \star \star \star} p<0.0001$. (F) CD31 staining of wound sites in every group at day 7 and day 14 post wounding. Scale bar: $100 \mu \mathrm{m}$. (G) Quantitation of average optical density (AOD) of wound sites in different groups ( $n=5$ per group). ${ }^{\star \star \star} p<0.001,{ }^{\star \star \star \star} p<0.0001$.

\section{Supplementary Files}

This is a list of supplementary files associated with this preprint. Click to download.

- Supplementaryinformationscrt.docx 